\title{
ON A CONVEXITY CONDITION IN NORMED LINEAR SPACES
}

BY

\author{
DANIEL P. GIESY
}

Introduction. The purpose of this paper is to study a convexity property on normed linear spaces (NLS's) which we call $B$-convexity. Interest in $B$-convexity is generated by a theorem of Anatole Beck ([1] or [2]) which states that a Banach space $\mathfrak{X}$ is $B$-convex if and only if a certain strong law of large numbers is valid for $\mathfrak{X}$-valued random variables.

Let $\mathfrak{X}$ be a NLS and $(S, \Sigma, m)$ a measure space. The Borel $\sigma$-field $\mathscr{B}$ of $\mathfrak{X}$ is the $\sigma$-field of sets generated by the subsets of $\mathfrak{X}$ open in the strong (norm) topology. A Borel set is an element of $\mathscr{B}$. A function $X$ from $S$ into $\mathfrak{X}$ is called strongly measurable if for each $B \in \mathscr{B}, X^{-1}(B)=\{s \in S: X(s) \in B\} \in \Sigma$. $X$ is called essentially separably valued if there is $N \in \Sigma, m(N)=0$, such that $X(S-N)$ is separable. If $\int_{S}\|X(s)\| d m(s)<\infty$ and $X$ is essentially separably valued, then $X$ is strongly (Bochner) integrable and there exists $y \in \mathfrak{Y}$, where $\mathfrak{Y}$ is the completion of $\mathfrak{X}$, such that for every $x^{*} \in \mathfrak{Y}^{*}$, the conjugate space to $\mathfrak{Y}, \int_{S} x^{*} X(s) d m(s)=x^{*} y$. In this case we write $y=\int_{S} X(s) d m(s)=\int_{S} X d m . y$ is called the strong integral of $X$.

A probability space is a measure space $(\Omega, \Sigma, \mathscr{P})$ of total measure $1(\mathscr{P}(\Omega)=1)$. An $\mathfrak{X}$-valued random variable on $\Omega$ is a function $X: \Omega \rightarrow \mathfrak{X}$ which is strongly measurable and essentially separably valued. The expectation of $X, E(X)=\int_{\Omega} X d \mathscr{P}$ if this integral exists. For a random variable $X$ with expectation, we define the variance of $X$ by

$$
\operatorname{var}(X)=\int_{\Omega}\|X(\omega)-E(X)\|^{2} d \mathscr{P}(\omega)
$$

We will call a finite set $X_{1}, \ldots, X_{n}$ of $\mathfrak{X}$-valued random variables on $\Omega$ independent if for each choice of Borel sets $B_{1}, \ldots, B_{n} \in \mathscr{B}$, we have

$$
\mathscr{P}\left(X_{1} \in B_{1} \& \cdots \& X_{n} \in B_{n}\right)=\mathscr{P}\left(X_{1} \in B_{1}\right) \cdots \mathscr{P}\left(X_{n} \in B_{n}\right)
$$

that is,

$$
\mathscr{P}\left\{\omega \in \Omega: X_{1}(\omega) \in B_{1} \& \cdots \& X_{n}(\omega) \in B_{n}\right\}=\prod_{i=1}^{n} \mathscr{P}\left\{\omega \in \Omega: X_{i}(\omega) \in B_{i}\right\} .
$$

An infinite set of random variables is independent if each finite subset is.

Presented to the Society in part November 30, 1963 under the title $A$ convexity condition on Banach spaces invariant under conjugation, April 25, 1964 under the title B-convexity, and September 3, 1965 under the title Fine structure of norms in B-convex spaces; received by the editors January $19,1966$. 
A NLS $\mathfrak{X}$ is said to satisfy property (A) if each independent sequence $\left\{X_{n}\right\}$ of $\mathfrak{X}$-valued random variables which satisfies the conditions that $E\left(X_{n}\right)=0$ for all $n$ and $\operatorname{var}\left(X_{n}\right)$ is uniformly bounded in $n$ also satisfies the condition that

$$
\lim _{n} n^{-1} \sum_{i=1}^{n} X_{i}=0
$$

strongly almost surely (i.e., $\lim _{n} n^{-1}\left\|\sum_{i=1}^{n} X_{i}(\omega)\right\|=0$ for almost all $\omega$ in $\Omega$ ). This is a form of the strong law of large numbers.

For $k \geqq 2$ and $\varepsilon>0, \mathfrak{X}$ is $k, \varepsilon$-convex ("convex of order $(k, \varepsilon)$ " in Beck's terminology) if for each choice of $x_{1}, \ldots, x_{k}$ from the unit ball of $\mathfrak{X}$,

$$
\left\| \pm x_{1} \pm x_{2} \pm \cdots \pm x_{k}\right\| \leqq k(1-\varepsilon)
$$

for some choice of the + and - signs. $\mathfrak{X}$ is $B$-convex (" $\mathfrak{X}$ satisfies property (B)" in Beck's terminology) if $\mathfrak{X}$ is $k, \varepsilon$-convex for some $k \geqq 2, \varepsilon>0$.

Beck's theorem then states that a Banach space $\mathfrak{X}$ satisfies property (A) if and only if $\mathfrak{X}$ is $B$-convex.

A great deal of what we will do here follows for general NLS's. Even in the case when a result follows on the hypothesis of topological completeness, this can sometimes be exorcised using the fact (see Remark I.2(c3)) that if $\mathfrak{X}$ is a $k, \varepsilon$-convex NLS and $\mathfrak{Y}$ is the completion of $\mathfrak{X}$, then $\mathfrak{Y}$ is $k, \varepsilon$-convex, and, of course $\mathfrak{Y}$ is a Banach space and contains an isometric copy of $\mathfrak{X}$. Thus, for example, Beck's theorem is valid for NLS's. The proof Beck gives that if $\mathfrak{X}$ is not $B$-convex, then $\mathfrak{X}$ does not satisfy property (A) (Beck [2, Theorem 11, p. 44]), is valid for general NLS's. For the converse, let $\mathfrak{X}$ be a $B$-convex NLS and let $\mathfrak{Y}$ be the completion of $\mathfrak{X}$. We may regard $\mathfrak{X}$ as a subspace of $\mathfrak{Y}$. Let $\left\{X_{n}\right\}$ be an independent sequence of $\mathfrak{X}$-valued random variables with $E\left(X_{n}\right)=0$ and $\operatorname{var}\left(X_{n}\right) \leqq M$ for all $n$. Then $\left\{X_{n}\right\}$ can be considered as an independent sequence of $\mathfrak{Y}$-valued random variables with the same properties, so by the Banach space version of Beck's theorem, $n^{-1} \sum_{i=1}^{n} X_{i} \rightarrow 0$ strongly almost surely in $\mathfrak{Y}$; hence, since $n^{-1} \sum_{i=1}^{n} X_{i}(\omega) \in \mathfrak{X}$ for each $\omega \in \Omega, n^{-1} \sum_{i=1}^{n} X_{i} \rightarrow 0$ strongly almost surely in $\mathfrak{X}$. Thus, Beck's theorem is valid for general NLS's.

For finite dimensional $\mathfrak{X}$, the strong law of large numbers can be verified by induction on the dimension on $\mathfrak{X}$ using the corresponding real- or complex-valued form of the strong law of large numbers. Thus, by Beck's theorem, finite dimensional spaces must be $B$-convex. In Example I.3(i) we verify directly that if $\operatorname{dim}(\mathfrak{X})<k$, then $X$ is $k, k^{-1}$-convex.

A forerunner of Beck's theorem, also due to Beck, stated that if $\mathfrak{X}$ was uniformly convex, then $\mathfrak{X}$ satisfied property (A). Thus by the stronger version, uniformly convex spaces must be $B$-convex. We verify directly that in fact uniformly convex 
spaces are 2, $\varepsilon$-convex for some $\varepsilon>0$. Beck tested two other generalizations of uniform convexity, namely locally uniform convexity and reflexivity, as hypotheses and found them lacking. In fact, he discovered an example of a locally uniformly convex, reflexive Banach space which did not satisfy property (A), hence is not $B$-convex (see Example I.7(i)).

We also verify that $\ell_{1}, \ell_{\infty}$, and $c_{0}$ are not $B$-convex (see Example I.3(iii) and (iv)). $B$-convexity can be viewed as a cancellation phenomenon. A NLS $\mathfrak{X}$ is $k, \varepsilon$ convex if for each choice of $x_{1}, \ldots, x_{k}$ from the unit ball of $X$, we may choose coefficients $\lambda_{1}, \ldots, \lambda_{k}$ from $\{+1,-1\}$ so that a uniformly great amount of cancellation occurs when we form the sum $x=\sum_{i=1}^{k} \lambda_{i} x_{i}$ so that $x$ fails by a factor of $\varepsilon$ to attain the maximum possible norm, $k$, for the sum of $k$ unit vectors. As a generalization of this for NLS's over the field of complex numbers, let $A$ be a subset of the boundary of the unit circle in the complex plane and call $\mathfrak{X} A$-convex if $\mathfrak{X}$ admits a cancellation phenomenon of the type just described with the set $\{+1,-1\}$, which we will hereinafter designate as $B$, replaced by $A$ (a rigorous definition of this is found in Definition I.1).

One might think that in an $A$-convex NLS $\mathfrak{X}$, the more vectors one takes, the more cancellation one could get. This is true and we get two results in this direction, a strong one using Beck's theorem (Theorem III.3; see also Comments III.4) and a weaker but extremely useful one (Lemma I.4) directly from the definitions, which state, in effect, that if $A$ is closed under multiplication (including the case $A=B$ ), then for every $\delta>0$ there exists $N$ such that if $n \geqq N$ and $x_{1}, \ldots, x_{n}$ are in the unit ball of $\mathfrak{X}$, there exist $\lambda_{1}, \ldots, \lambda_{n} \in A$ such that $\left\|\sum_{i=1}^{n} \lambda_{i} x_{i}\right\| \leqq n \delta$.

Using this result, we prove in Theorem I.5 that if $A$ contains at least 2 elements, then a NLS is $A$-convex if and only if it is $B$-convex.

We find a characterization of non- $B$-convex spaces (Lemma I.6) which has a geometric interpretation; namely $\mathfrak{X}$ is not $B$-convex exactly in case there are arbitrarily good copies of finite dimensional $\ell_{1}$ in $\mathfrak{X}$ for arbitrarily large finite dimension.

$\S$ II is devoted to studying how $B$-convexity behaves under common operations on NLS's. We get results on first and second conjugates and factor spaces of $B$-convex spaces, and on the image of $B$-convex spaces under continuous linear transformations. Finite and infinite direct sums of $B$-convex spaces are studied.

§III contains results about internal properties of $B$-convex spaces. We sharpen one direction of Beck's theorem and apply this result to investigate the cancellation phenomenon in $B$-convex spaces. We find a condition under which a $B$-convex space is reflexive. We give an example of a space with several properties of a uniformly convex space without being uniformly convex.

$\S I V$ gives open questions, partial results, and acknowledgements.

An internal reference in this paper which does not include a section number refers to the section in which it occurs. 
I. Preliminaries. In this section we give the basic definitions and some examples and we derive some technical results about $B$-convex and non- $B$-convex spaces.

1. Definitions. Let $B=\{1,-1\}, C=\{z: z$ is a complex number with $|z|=1\}$. Let $\mathfrak{X}$ be a normed linear space (NLS) over the scalar field $\Phi$, where $\Phi$ is the real or complex numbers. As a notational convenience, we let $D(=D(\mathfrak{X}, \Phi))$ be $B$ or $C$ according as $\mathfrak{X}$ is a real or complex NLS respectively. Let $S=\{x \in \mathfrak{X}:\|x\| \leqq 1\}$. Let $A \subset D$. Define $a_{k}(\mathfrak{X}, A)=\sup \left(\inf k^{-1}\left\|\sum_{i=1}^{k} \lambda_{i} x_{i}\right\|\right)$ where the inf is taken over all sequences $\left\{\lambda_{i}\right\}_{1}^{k} \in A^{k}$ and the sup is taken over all sequences $\left\{x_{i}\right\}_{1}^{k} \in S^{k}$. Thus, $a_{k}(\mathfrak{X}, A)$ measures the amount of cancellation one can be sure of getting by fitting $k$ vectors from the unit ball of $\mathfrak{X}$ with coefficients from the set $A$. If the set $A$ is closed (in particular, if $A$ is finite), then for each sequence $x_{1}, \ldots, x_{k}$ the inf is achieved for some choice of $\lambda_{1}, \ldots, \lambda_{k}$, so the following definition agrees with the one given in the introduction in the case $A=B$. $\mathfrak{X}$ is $A, k, \varepsilon$-convex if $a_{k}(\mathfrak{X}, A) \leqq 1-\varepsilon$. $\mathfrak{X}$ is $A$-convex if there exists $k \geqq 2, \varepsilon>0$ such that $\mathfrak{X}$ is $A, k, \varepsilon$-convex. For the frequently used " $B, k$, $\varepsilon$-convex" we write " $k, \varepsilon$-convex."

2. Remarks. (a) It is immediate from the triangle inequality that $a_{k}(\mathfrak{X}, A) \leqq 1$.

(b) If $A=\{\lambda\}$, a single point, then $a_{k}(\mathfrak{X}, A)=1$ for all $k$ as can be seen by considering the sequence $x_{1}=x_{2}=\cdots=x_{k}=x$ for any $x$ with $\|x\|=1$. Then

$$
a_{k}(\mathfrak{X}, A) \geqq \inf k^{-1}\left\|\sum_{i=1}^{k} \lambda_{i} x_{i}\right\|=k^{-1}\left\|\sum_{i=1}^{k} \lambda x\right\|=k^{-1}\|k \lambda x\|=k^{-1} k|\lambda| \cdot\|x\|=1 .
$$

Henceforth we will assume that all sets $A$ contain at least two points. In the real case, $B$ is the only such set.

(c) If $\mathfrak{X}_{2} \subseteq \mathfrak{X}_{1}$ and $A_{1} \subseteq A_{2}$, then $a_{k}\left(\mathfrak{X}_{2}, A_{2}\right) \leqq a_{k}\left(\mathfrak{X}_{1}, A_{1}\right)$ since in calculating $a_{k}\left(\mathfrak{X}_{2}, A_{2}\right)$, the inf extends over a larger set and the sup over a smaller set than in calculating $a_{k}\left(\mathfrak{X}_{1}, A_{1}\right)$. Further, if $\mathfrak{X}_{2}$ is dense in $\mathfrak{X}_{1}$ (including $\mathfrak{X}_{2}=\mathfrak{X}_{1}$ ) and $A_{1}$ is dense in $A_{2}$ (including $\left.A_{1}=A_{2}\right), a_{k}\left(\mathfrak{X}_{2}, A_{2}\right)=a_{k}\left(\mathfrak{X}_{1}, A_{1}\right)$ since any element of the form $\sum_{i=1}^{k} \lambda_{i} x_{i}$ with $\lambda_{i} \in A_{2}, x_{i} \in \mathfrak{X}_{2},\left\|x_{i}\right\| \leqq 1$ can be approximated arbitrarily well by an element of the form $\sum_{i=1}^{k} \mu_{i} y_{i}$ with $\mu_{i} \in A_{1}$ and $y_{i} \in \mathfrak{X}_{1},\left\|y_{i}\right\| \leqq 1$, and vice versa. Hence, if $\delta \leqq \varepsilon$ and $\mathfrak{X}_{1}$ is $A_{1}, k, \varepsilon$-convex ( $A_{1}$-convex) then $\mathfrak{X}_{2}$ is $A_{2}, k, \delta$ convex ( $A_{2}$-convex).

Particularly useful special cases of the above include:

(c1) If $\mathfrak{X}$ is $A, k, \varepsilon$-convex ( $A$-convex) and $\mathfrak{Y} \subseteq \mathfrak{X}$, then $\mathfrak{Y}$ is $A, k$, $\varepsilon$-convex $(A$ convex).

(c2) If $\mathfrak{X}$ is $A, k, \varepsilon$-convex and $\delta \leqq \varepsilon$, then $\mathfrak{X}$ is $A, k, \delta$-convex.

(c3) If $\mathfrak{Y} \subseteq \mathfrak{X}$ and $\mathfrak{Y}$ is $A, k, \varepsilon$-convex ( $A$-convex) then $\mathrm{cl} \mathfrak{Y}$ is $A, k, \varepsilon$-convex ( $A$-convex). In particular, if $\mathfrak{X}$ is the completion of $\mathfrak{Y}$, cl $\mathfrak{Y}=\mathfrak{X}$, so $\mathfrak{X}$ is $A, k, \varepsilon$ convex ( $A$-convex).

3. ExAmples. Most of the following examples will be stated in terms of $B$ convexity or sometimes in the complex case $C$-convexity. In the light of the following Theorem 5, this is no real loss of generality. 
(i) All finite dimensional spaces are $D$-convex. In fact, if the dimension of $\mathfrak{X}$ is less than $k$, then $\mathfrak{X}$ is $D, k, k^{-1}$-convex. Let $x_{1}, \ldots, x_{k} \in S$. We may choose scalars $a_{1}, \ldots, a_{k}$ not all zero and normalized so that $\sup _{i}\left|a_{i}\right|=1$ such that $\sum_{i=1}^{k} a_{i} x_{i}=0$. Let $\lambda_{i}=\operatorname{sg}\left(a_{i}\right)$ where $\operatorname{sg}(\cdot)$ is the sign function defined by $\operatorname{sg}(a)=a /|a|$ if $a \neq 0, \operatorname{sg}(0)=1$. Then $\lambda_{i} \in D,\left|\lambda_{i}-a_{i}\right| \leqq 1$ for all $i$, and $\lambda_{i}-a_{i}=0$ for at least one $i, 1 \leqq i \leqq k$. Hence

$$
\begin{aligned}
k^{-1}\left\|\sum_{i=1}^{k} \lambda_{i} x_{i}\right\| & =k^{-1}\left\|\sum_{i=1}^{k} a_{i} x_{i}+\sum_{i=1}^{k}\left(\lambda_{i}-a_{i}\right) x_{i}\right\| \\
& \leqq k^{-1} \sum_{i=1}^{k}\left|\lambda_{i}-a_{i}\right| \leqq k^{-1}(k-1)=1-k^{-1} .
\end{aligned}
$$

Therefore $\mathfrak{X}$ is $D, k, k^{-1}$-convex.

(ii) All uniformly convex spaces are $B$-convex. In fact, a uniformly convex space is $2, \varepsilon$-convex for suitable $\varepsilon$. A NLS $\mathfrak{X}$ is uniformly convex if for each $\varepsilon>0$ there exists $\delta(\varepsilon)>0$ such that if $\|x\|=\|y\|=1$ and $\|x-y\| \geqq \varepsilon$ then $\|x+y\|<2(1-\delta(\varepsilon))$. This condition says that if two unit vectors are $\varepsilon$ apart, they fail by $2 \delta$ to add up to the maximum possible norm of 2 , and this occurs uniformly over the entire surface of the unit ball of $\mathfrak{X}$. Equivalently (see Lemma II.15), $\mathfrak{X}$ is uniformly convex if for each $\varepsilon>0$ there exists $\delta^{\prime}>0$ such that if $x, y \in S$ with $\|x-y\|>\varepsilon$, then $\|x+y\| \leqq 2\left(1-\delta^{\prime}\right)$. Let $\eta$ be the $\delta^{\prime}$ corresponding to $\varepsilon=1$. Define $\theta=\min (1 / 2, \eta)$. $\mathfrak{X}$ is $2, \theta$-convex, for if $x, y \in S$, then either $\|x-y\| \leqq 1=2(1-1 / 2) \leqq 2(1-\theta)$ or $\|x-y\|>1$ in which case $\|x+y\| \leqq 2(1-\eta) \leqq 2(1-\theta)$.

Examples of uniformly convex spaces include (finite or infinite dimensional) $\ell_{p}$ and $L_{p}, 1<p<\infty$, (cf. Clarkson [3]) and all inner product spaces. The Jordanvon Neumann characterization of inner product spaces [14] gives us

$$
\|x+y\|^{2}+\|x-y\|^{2}=2\left(\|x\|^{2}+\|y\|^{2}\right),
$$

so if $\|x\|=\|y\|=1$, then $\|x+y\|^{2}+\|x-y\|^{2}=4$. Hence if $\|x+y\| \geqq \varepsilon>0$, then $\|x-y\| \leqq\left(4-\varepsilon^{2}\right)^{1 / 2}=2(1-\delta(\varepsilon))$ for $\delta(\varepsilon)=1-\left(1-(\varepsilon / 2)^{2}\right)^{1 / 2}>0$.

(iii) $\ell_{1}$ is not $A$-convex. In some sense, $\ell_{1}$ seems to be the least $A$-convex of any imaginable space. Let $x_{n}=\left\{\delta_{n i}\right\}$, the $n$th unit vector in $\ell_{1}$. Let $\lambda_{1}, \ldots, \lambda_{k} \in A$. Then $\left|\lambda_{i}\right|=1$.

$$
\begin{aligned}
k^{-1}\left\|\sum_{i=1}^{k} \lambda_{i} x_{i}\right\| & =k^{-1}\left\|\left(\lambda_{1}, \lambda_{2}, \ldots, \lambda_{k}, 0, \ldots\right)\right\| \\
& =k^{-1} \sum_{i=1}^{k}\left|\lambda_{i}\right|=1 .
\end{aligned}
$$

Hence $a_{k}\left(\ell_{1}, A\right)=1$ for all $k$ and $\ell_{1}$ is not $A$-convex. 
(iv) $\ell_{\infty}$ and $c_{0}$ are not $B$-convex. Here, $\ell_{\infty}$ is the space of all bounded sequences of scalars under the norm $\|x\|=\sup _{n}\left|x_{n}\right| . c_{0}$ is the subspace of $\ell_{\infty}$ of all sequences convergent to 0 . Fix $k$. Let

$$
\begin{aligned}
& x_{1}=(+1,-1,+1,-1, \ldots,+1,-1,0,0, \ldots) \\
& x_{2}=(+1,+1,-1,-1, \ldots,-1,-1,0,0, \ldots) \\
& \vdots \\
& x_{k}=(+1,+1,+1,+1, \ldots,-1,-1,0,0, \ldots)
\end{aligned}
$$

where in each vector there are $2^{k}$ nonzero terms and the nonzero terms in $x_{i}$ consist of alternating blocks of +1 's and -1 's, each block of length $2^{i-1}$. Note that $x_{i} \in c_{0}$. Denoting the $j$ th coordinate of $x_{i}$ by $x_{i}(j)$, if $\xi_{1}, \ldots, \xi_{k}$ is a sequence of +1 's and -1 's, there is a $j$ such that $\xi_{i}=x_{i}(j)$ for all $i$. Thus, the $j$ th coordinate of $\sum_{i=1}^{k} \xi_{i} x_{i}$ is $k$ so $\left\|\sum_{i=1}^{k} \xi_{i} x_{i}\right\|=k$. Thus $a_{k}\left(c_{0}, B\right)=a_{k}\left(\ell_{\infty}, B\right)=1$, so $c_{0}$ and $\ell_{\infty}$ are not $B$-convex.

4. Lemma. Let $A$ be closed under multiplication. Let $\mathfrak{X}$ be a NLS. Then either $a_{k}(\mathfrak{X}, A)=1$ for all $k$ (in which case $\mathfrak{X}$ is not $A$-convex) or $\lim _{k} a_{k}(\mathfrak{X}, A)=0$ (in which case $\mathfrak{X}$ is $A$-convex). In the latter case there exists $\gamma>0$ such that $a_{k}(\mathfrak{X}, A)=O\left(k^{-\gamma}\right)$.

REMARKS ON THIS LEMMA. This is the first result of the form "the more vectors one takes, the more cancellation one gets." Using this lemma in its present form and the following Theorem 5, one can reach the same conclusion under the hypothesis that the closure of $A$ has some nontrivial (i.e., more than one point) subset $A^{\prime}$ which can be rotated to obtain a set $A^{\prime \prime}$ which is closed under multiplication. A necessary condition on the set $A$ that $\lim a_{k}(\mathfrak{X}, A)=0$ is that the closed convex hull of $A$ in the complex plane contain the origin, for otherwise we could separate $A$ from the origin by a line at a distance $\delta>0$ from the origin. Then $\lambda_{1}, \ldots, \lambda_{k} \in A$ implies $\left|\sum_{i=1}^{k} \lambda_{i}\right| \geqq k \delta$. Letting $x \in X,\|x\|=1$,

$$
\begin{aligned}
a_{k}(\mathfrak{X}, A) & \geqq \inf _{\left\{\lambda_{i}\right\} \subset A} k^{-1}\left\|\sum_{i=1}^{k} \lambda_{i} x\right\|=\inf k^{-1}\left|\sum_{i=1}^{k} \lambda_{i}\right| \\
& \geqq \inf k^{-1} \cdot k \delta=\delta>0
\end{aligned}
$$

so $\lim _{k} a_{k}(\mathfrak{X}, A)$ could not be 0 . We show in Corollary III.5 that this necessary condition on $\mathrm{A}$ is also sufficient.

Proof of Lemma. If $\mathfrak{X}$ is not $A$-convex, then by definition, $a_{k}(\mathfrak{X}, A)=1$ for all $k$.

Now suppose $\mathfrak{X}$ is $A$-convex. Then there exist $k \geqq 2, \varepsilon>0$ such that $\mathfrak{X}$ is $A, k, 2 \varepsilon$ convex. We may pick $\varepsilon<1-k^{-1}$, so $k(1-\varepsilon)>1$. Then for any $k$ vectors $x_{1}, \ldots$, $x_{k} \in S$, inf $\left\{k^{-1}\left\|\sum_{i=1}^{k} \lambda_{i} x_{i}\right\|: \lambda_{i} \in A\right\} \leqq 1-2 \varepsilon$ so we may pick $\lambda_{1}, \ldots, \lambda_{k} \in A$ such 
that $\left\|\sum_{i=1}^{k} \lambda_{i} x_{i}\right\| \leqq k(1-\varepsilon)$. The basic idea of this proof can be seen in the following special case. We pick $k^{2}$ vectors $x_{1}, \ldots, x_{k^{2}}$ from $S$. Grouping them into $k$ sets of $k$ vectors each, we fit each set with elements of $A$ so that the resulting sum has norm $\leqq k(1-\varepsilon)$. Then each sum can be multiplied by an element of $A$ so that they add to a vector of norm $\leqq k^{2}(1-\varepsilon)^{2}$. Thus, let $\lambda_{i}^{\prime} \in A$ be chosen so that if $y_{j}=$ $\sum_{i=k(j-1)+1}^{k j} \lambda_{i} x_{i}$, then $\left\|y_{j}\right\| \leqq k(1-\varepsilon)$ for $j=1,2, \ldots, k$. Then pick $\lambda_{j}^{\prime \prime} \in A$ so that $\left\|\sum_{j=1}^{k} \lambda_{j}^{\prime \prime} y_{j}\right\| \leqq k^{2}(1-\varepsilon)^{2}$. For $k(j-1)+1 \leqq i \leqq k j$, let $\lambda_{i}=\lambda_{i}^{\prime} \lambda_{j}^{\prime \prime}$. Then $\lambda_{i} \in A$ for all $i$. So

$$
\begin{aligned}
\left\|\sum_{j=1}^{k} \lambda_{j}^{\prime \prime} y_{j}\right\| & =\left\|\sum_{j=1}^{k} \lambda_{j}^{\prime \prime} \sum_{i=k(j-1)+1}^{k j} \lambda_{i}^{\prime} x_{i}\right\| \\
& =\left\|\sum_{i=1}^{k^{2}} \lambda_{i} x_{i}\right\| \leqq k^{2}(1-\varepsilon)^{2} .
\end{aligned}
$$

So having chosen $x_{1}, \ldots, x_{k^{2}} \in S$, we can find $\lambda_{i} \in A$ so that

$$
\left\|\sum_{i=1}^{k^{2}} \lambda_{i} x_{i}\right\| \leqq k^{2}(1-\varepsilon)^{2} .
$$

By induction, we easily see that we can repeat the process so that if $x_{1}, \ldots, x_{k^{m}} \in S$, we can find $\lambda_{i} \in A$ so that $\left\|\sum_{i=1}^{k^{m}} \lambda_{i} x_{i}\right\| \leqq k^{m}(1-\varepsilon)^{m}$.

Now for arbitrary $n$, write $n=a_{m} k^{m}+\cdots+a_{1} k+a_{0}, 0 \leqq a_{i} \leqq k-1$ for $0 \leqq i \leqq m$, $a_{m} \neq 0$. Let $x_{1}, \ldots, x_{n} \in S$. Group these vectors into $a_{m}$ sets of $k^{m}$ vectors, $a_{m-1}$ sets of $k^{m-1}$ vectors, . ., $a_{1}$ sets of $k$ vectors and $a_{0}$ single vectors. Fit each set of $k^{i}$ vectors with elements of $A$ so that the resulting sum has norm $\leqq k^{i}(1-\varepsilon)^{i}$. By using the triangle inequality, we find that we have found $\lambda_{i} \in A$ such that

$$
\left\|\sum_{i=1}^{n} \lambda_{i} x_{i}\right\| \leqq \sum_{j=1}^{m} a_{j} k^{j}(1-\varepsilon)^{j} .
$$

Then since $a_{j} \leqq k-1$ for all $j$ and $n \geqq k^{m}$ so $n^{-1} \leqq k^{-m}$,

$$
\begin{aligned}
n^{-1}\left\|\sum_{i=1}^{n} \lambda_{i} x_{i}\right\| & \leqq k^{-m} \sum_{j=1}^{m}(k-1) k^{\jmath}(1-\varepsilon)^{\jmath} \\
& =k^{-m}(k-1)\left(\frac{k^{m+1}(1-\varepsilon)^{m+1}-1}{k(1-\varepsilon)-1}\right) \\
& \leqq\left(\frac{k(k-1)(1-\varepsilon)}{k(1-\varepsilon)-1}\right)(1-\varepsilon)^{m} \text { since } k(1-\varepsilon)>1 \\
& \leqq K(1-\varepsilon)^{\log _{k} n} \text { since } m \leqq \log _{k} n \\
& =K n^{-\gamma} \text { for } \gamma=-\ln (1-\varepsilon) / \ln k>0 .
\end{aligned}
$$


Note that the constants $K$ and $\gamma$ depend only on $k$ and $\varepsilon$, not on the sequence $\left\{x_{i}\right\}$ or even on $\mathfrak{X}$. Thus $\inf _{\lambda_{i} \in A} n^{-1}\left\|\sum_{i=1}^{n} \lambda_{i} x_{i}\right\| \leqq K n^{-\gamma}$ for all sequences $x_{1}, \ldots$, $x_{n} \in S$, hence $a_{n}(\mathfrak{X}, A)=O\left(n^{-\gamma}\right) \rightarrow 0$ as $n \rightarrow \infty$. Q.E.D.

5. TheOREM. If $A_{1}$ and $A_{2}$ both contain at least two points, $\mathfrak{X}$ is $A_{1}$-convex if and only if $\mathfrak{X}$ is $A_{2}$-convex.

Proof. If $\mathfrak{X}$ is a real NLS, the conditions imply $A_{1}=A_{2}=B$ and the result is trivial. Suppose $\mathfrak{X}$ is a complex NLS. The proof proceeds in four steps.

(a) If $\mathfrak{X}$ is $A_{1}$-convex, $\mathfrak{X}$ is $C$-convex (Remark 2 (c)).

(b) If $\mathfrak{X}$ is $C$-convex, $\mathfrak{X}$ is $P$-convex where $P=\left\{\omega_{n}=\exp ((2 \pi n / 5) i): 0 \leqq n \leqq 4\right\}$, the complex fifth roots of unity. For each $\lambda \in C$ choose $\omega(\lambda)$ to be a fifth root of unity closest to $\lambda$. Then the short arc of the unit circle joining $\lambda$ and $\omega(\lambda)$ is at most $\pi / 5$ long so $|\lambda-\omega(\lambda)| \leqq \pi / 5<3 / 4$ (say). Since $C$ is closed under multiplication we may choose $k$ so large that for each choice of $x_{1}, \ldots, x_{k} \in S$ there are $\lambda_{1}, \ldots$, $\lambda_{k} \in C$ such that $k^{-1}\left\|\sum_{i=1}^{k} \lambda_{i} x_{i}\right\| \leqq 1 / 8$ (Lemma 4). This choice is such that if we replace each $\lambda_{i}$ in the above sum by its best approximation in $P$, we still have cancellation. Thus

$$
\begin{aligned}
k^{-1}\left\|\sum_{i=1}^{k} \omega\left(\lambda_{i}\right) x_{i}\right\| & \leqq k^{-1}\left\|\sum_{i=1}^{k} \lambda_{i} x_{i}\right\|+k^{-1}\left\|\sum_{i=1}^{k}\left(\omega\left(\lambda_{i}\right)-\lambda_{i}\right) x_{i}\right\| \\
& \leqq 1 / 8+k^{-1} \sum_{i=1}^{k}\left|\omega\left(\lambda_{i}\right)-\lambda_{i}\right|\left\|x_{i}\right\| \\
& \leqq 1 / 8+k^{-1} \sum_{j=1}^{k}(3 / 4) \cdot 1=7 / 8 .
\end{aligned}
$$

So $a_{k}(\mathfrak{X}, P) \leqq 7 / 8$ and $\mathfrak{X}$ is $P, k, 1 / 8$-convex, hence $P$-convex.

(c) If $\mathfrak{X}$ is $P$-convex, $\mathfrak{X}$ is $B$-convex. The proof here follows the same idea as the previous one of getting a lot of cancellation using coefficients from $P$, then approximating these with \pm 1 . However, since $\left|\omega_{1}-1\right|=\left|\omega_{4}-1\right|=2 \sin \pi / 5>1$, we must arrange before approximating the coefficients from $P$ with \pm 1 that at most $2 / 5$ of them are $\omega_{1}$ and $\omega_{4}$. Then the remaining at least $3 / 5$ are at a distance at most. $2 \sin \pi / 10$ from their best approximations with \pm 1 . If we let

$$
\delta_{1}=(2 / 5) 2 \sin \pi / 5+(3 / 5) 2 \sin \pi / 10
$$

then $.80 \leqq \delta_{1} \leqq .82$. Choose $\delta_{2}>0$ and $\varepsilon>0$ so that $\delta_{1}+\delta_{2}=1-\varepsilon<1$. Using Lemma 4 , choose $k$ so large that for any choice of $x_{1}, \ldots, x_{k} \in S$ there are $\zeta_{1}^{\prime}, \ldots, \zeta_{k}^{\prime} \in P$ such that $k^{-1}\left\|\sum_{i=1}^{k} \zeta_{i}^{\prime} x_{i}\right\| \leqq \delta_{2}$. A simple combinatorial argument yields the fact that for some choice of $n$, at most $2 k / 5$ of the $\zeta_{i}^{\prime}$ are $\omega_{n+1}$ and $\omega_{n-1}$ (all subscripts 
on $\omega$ 's reduced modulo 5). Letting $\zeta_{i}=\omega_{-n} \zeta_{i}^{\prime}$, we see that $k^{-1}\left\|\sum_{i=1}^{k} \zeta_{i} x_{i}\right\| \leqq \delta_{2}$ and at most $2 k / 5$ of the $\zeta_{i}$ are $\omega_{1}$ and $\omega_{4}$.

If $\zeta_{i}=\omega_{0}, \omega_{1}$ or $\omega_{4}$, let $\lambda_{i}=1$ while if $\zeta_{i}=\omega_{2}$ or $\omega_{3}$, let $\lambda_{i}=-1$. Then for at most $2 k / 5$ of the $i,\left|\zeta_{i}-\lambda_{i}\right|=2 \sin \pi / 5$ while for at least $3 k / 5$ of the $i,\left|\zeta_{i}-\lambda_{i}\right| \leqq 2 \sin \pi / 10$. Thus

$$
k^{-1} \sum_{i=1}^{k}\left|\zeta_{i}-\lambda_{i}\right| \leqq(2 / 5) 2 \sin \pi / 5+(3 / 5) 2 \sin \pi / 10=\delta_{1}
$$

Hence

$$
\begin{aligned}
k^{-1}\left\|\sum_{i=1}^{k} \lambda_{i} x_{i}\right\| & \leqq k^{-1}\left\|\sum_{i=1}^{k}\left(\zeta_{i}-\lambda_{i}\right) x_{i}\right\|+k^{-1}\left\|\sum_{i=1}^{k} \zeta_{i} x_{i}\right\| \\
& \leqq k^{-1} \sum_{i=1}^{k}\left|\zeta_{i}-\lambda_{i}\right|+\delta_{2} \\
& \leqq \delta_{1}+\delta_{2}=1-\varepsilon
\end{aligned}
$$

Thus $a_{k}(\mathfrak{X}, B) \leqq 1-\varepsilon$ so $\mathfrak{X}$ is $k, \varepsilon$-convex, hence $B$-convex.

(d) If $\mathfrak{X}$ is $B$-convex, then $\mathfrak{X}$ is $A_{2}$-convex. Choose $\lambda, \mu \in A_{2}, \lambda \neq \mu$. Then there are scalars $\alpha, \beta$ such that $\lambda=\alpha+\beta, \mu=\alpha-\beta,|\alpha|<1, \beta \neq 0$. Pick $\delta, 0<\delta<1$, and $\varepsilon>0$ so that $|\alpha|+|\beta|(1-\delta)=1-\varepsilon<1$. Pick $k$ so large that for $x_{1}, \ldots, x_{k} \in S$ there are $\xi_{1}, \ldots, \xi_{k} \in B$ so that $k^{-1}\left\|\sum_{i=1}^{k} \xi_{i} x_{i}\right\|<1-\delta$. Then $\alpha+\xi_{i} \beta \in A_{2}$ for each $i$ and

$$
\begin{aligned}
k^{-1}\left\|\sum_{i=1}^{k}\left(\alpha+\xi_{i} \beta\right) x_{i}\right\| & \leqq k^{-1}|\alpha|\left\|\sum_{i=1}^{k} x_{i}\right\|+k^{-1}|\beta|\left\|\sum_{i=1}^{k} \xi_{i} x_{i}\right\| \\
& \leqq k^{-1}|\alpha| k+|\beta|(1-\delta) \\
& =1-\varepsilon .
\end{aligned}
$$

Thus $\mathfrak{X}$ is $A_{2}, k, \varepsilon$-convex, hence $A_{2}$-convex. Q.E.D.

REMARKS. We now see that all nontrivial forms of $A$-convexity are equivalent to $B$-convexity, and in what follows we shall restrict our attention to $B$-convexity unless some special insight can be gained by using the more general form. Beck has proven independently the following (unpublished) theorem: If $B_{n}$ denotes the $n n$th roots of unity, $\mathfrak{X}$ satisfies property (A) if and only if $\mathfrak{X}$ is $B_{n^{-}}$convex. This showed that $\mathfrak{X}$ was $B\left(=B_{2}\right)$-convex if and only if $\mathfrak{X}$ was $B_{n}$-convex. An argument similar to part (b) of the above proof yielded "If $\mathfrak{X}$ is $C$-convex, then $\mathfrak{X}$ is $B_{n}$-convex for sufficiently large $n$ " even without recourse to Lemma 4 . The present theorem yields the following generalization of Beck's theorem: If $A \subset D$ has at least two points, then $\mathfrak{X}$ is $A$-convex if and only if $\mathfrak{X}$ satisfies property (A). 
6. Lemma. A NLS $\mathfrak{X}$ is not $D$-convex if and only if for every $k \geqq 2, \varepsilon>0$, there exist $x_{1}, \ldots, x_{k} \in S$ such that for each choice of scalars $\alpha_{1}, \ldots, \alpha_{k}$,

$$
(1-\varepsilon) \sum_{i=1}^{k}\left|\alpha_{i}\right| \leqq\left\|\sum_{i=1}^{k} \alpha_{i} x_{i}\right\| \leqq \sum_{i=1}^{k}\left|\alpha_{i}\right| .
$$

Remarks. Geometrically, this lemma states that NLS which is not $D$-convex contains arbitrarily good approximations of $k$-dimensional $\ell_{1}$ over the appropriate scalar field for all $k$.

The idea of the proof is this: Given $k$ and $\varepsilon$, we choose $x_{1}, \ldots, x_{k}$ appropriately using the failure of $\mathfrak{X}$ to be $D$-convex. Then in each "quadrant" of the span of $x_{1}, \ldots, x_{k}$ (we illustrate with the positive "quadrant") the surface of the unit sphere comes near or touches the convex hull of $x_{1}, \ldots, x_{k}$ at each of the points $x_{i}$, and comes very near the center of that convex hull at $k^{-1}\left(x_{1}+x_{2}+\cdots+x_{k}\right)$. Since the unit sphere is convex, its surface cannot get very far from the convex hull of $x_{1}, \ldots, x_{k}$ anywhere in the positive "quadrant." If the span of $x_{1}, \ldots, x_{k}$ is renormed with the $\ell_{1}$ norm using $x_{1}, \ldots, x_{k}$ as basis vectors, the convex hull of $x_{1}, \ldots, x_{k}$ lies in the surface of the $\ell_{1}$ unit sphere.

Proof. Suppose the latter condition of the theorem holds. Given $k$ and $\varepsilon$, let $x_{1}, \ldots, x_{k}$ be the vectors given by that condition. Choose arbitrary scalars $\lambda_{1}, \ldots, \lambda_{k} \in D$. Then

$$
\begin{aligned}
\left\|\sum_{i=1}^{k} \lambda_{i} x_{i}\right\| & \geqq(1-\varepsilon) \sum_{i=1}^{k}\left|\lambda_{i}\right| \\
& =k(1-\varepsilon) .
\end{aligned}
$$

Hence $a_{k}(\mathfrak{X}, D) \geqq 1-\varepsilon$. Since this is true for all $k$ and $\varepsilon, \mathfrak{X}$ is not $D$-convex.

Now suppose $\mathfrak{X}$ is not $D$-convex. Pick $k \geqq 2, \varepsilon>0$. We may suppose $\varepsilon<1$, or else the desired conclusion holds trivially for any choice of $k$ vectors from $S$. Let $\delta=k^{-1} \varepsilon$. Using the fact that $\mathfrak{X}$ is not $D$-convex, hence not $D, k, \delta$-convex, we may choose $x_{1}, \ldots, x_{k} \in S$ so that for any choice of $\lambda_{1}, \ldots, \lambda_{k} \in D$,

$$
k^{-1}\left\|\sum_{i=1}^{k} \lambda_{i} x_{i}\right\|>1-\delta
$$

The linear span of $\left\{x_{1}, \ldots, x_{k}\right\}$ is not $D, k, \delta$-convex. Any space of dimension less than $k$ is $D, k, k^{-1}$-convex (Example 3(i)), and $\delta<k^{-1}$, so the linear span of $\left\{x_{1}, \ldots, x_{k}\right\}$ must be $k$-dimensional. Hence $x_{1}, \ldots, x_{k}$ are linearly independent. Choose scalars $\alpha_{1}, \ldots, \alpha_{k} .\left\|\sum_{i=1}^{k} \alpha_{i} x_{i}\right\| \leqq \sum_{i=1}^{k}\left|\alpha_{i}\right| \cdot\left\|x_{i}\right\| \leqq \sum_{i=1}^{k}\left|\alpha_{i}\right|$, giving us onehalf of the required inequality. Since $x_{1}, \ldots, x_{k}$ are independent, $\sum_{i=1}^{k} \alpha_{i} x_{i}=0$ if and only if $\alpha_{i}=0$ for all $i$, if and only if $\sum_{i=1}^{k}\left|\alpha_{i}\right|=0$. In this case

$$
0=(1-\varepsilon) \sum_{i=1}^{k}\left|\alpha_{i}\right| \leqq\left\|\sum_{i=1}^{k} \alpha_{i} x_{i}\right\|=0 \text {. }
$$


Now suppose $\alpha=\sum_{i=1}^{k}\left|\alpha_{i}\right| \neq 0$ so $\lambda=\left\|\sum_{i=1}^{k} \alpha_{i} x_{i}\right\| \neq 0$. We wish to show $(1-\varepsilon) \alpha \leqq \lambda$. As before, let $\operatorname{sg}(0)=1, \operatorname{sg}(\xi)=\xi /|\xi|$ for $\xi \neq 0$. Then $\operatorname{sg}(\xi) \in D$ for each scalar $\xi$. Let $\beta_{i}=\left|\alpha_{i}\right| \lambda^{-1}$ and $y_{i}=\operatorname{sg}\left(\alpha_{i}\right) x_{i}$. Then $\beta=\sum_{i=1}^{k} \beta_{i}=\lambda^{-1} \sum_{i=1}^{k}\left|\alpha_{i}\right|=\lambda^{-1} \alpha$ and $\left\|\sum_{i=1}^{k} \beta_{i} y_{i}\right\|=\left\|\sum_{i=1}^{k} \lambda^{-1} \alpha_{i} x_{i}\right\|=1$ so we must show that $\beta \leqq(1-\varepsilon)^{-1}$. Suppose $\beta>(1-\varepsilon)^{-1}$. Then $\beta>(1-\delta)^{-1}$ since $\delta<\varepsilon$. Note that

$$
\left\|\sum_{i=1}^{k} k^{-1}(1-\delta)^{-1} y_{i}\right\|=k^{-1}(1-\delta)^{-1}\left\|\sum_{i=1}^{k} \operatorname{sg}\left(\alpha_{i}\right) x_{i}\right\|>1
$$

by the choice of the $x_{i}$.

We now restrict our attention to the real span of $\left\{y_{1}, \ldots, y_{k}\right\}$. The hyperplane $H$ through $y_{1}, \ldots, y_{k}$ is the set of all points of the form $\sum_{i=1}^{k} \gamma_{i} y_{i}$ where $\sum_{i=1}^{k} \gamma_{i}=1$. The convex hull $H^{\prime}$ of $y_{1}, \ldots, y_{k}$ is the set of all points of the form $\sum_{i=1}^{k} \gamma_{i} y_{i}$ where $\sum_{i=1}^{k} \gamma_{i}=1$ and $\gamma_{i} \geqq 0$ for all $i$. Since $\left\|y_{i}\right\| \leqq 1$ for all $i, H^{\prime} \subset S$.

The line through $u=\sum_{i=1}^{k} \beta_{i} y_{i}$ and $v=\sum_{i=1}^{k} k^{-1}(1-\delta)^{-1} y_{i}$ is the set of all points of the form $(1-\gamma) u+\gamma v$ for $\gamma$ real. This line intersects $H$ at $w=(1-\gamma) u+\gamma v$ for

$$
\gamma=\frac{\beta-1}{\beta-(1-\delta)^{-1}}
$$

We see $\gamma>1$ because $\beta>(1-\varepsilon)^{-1}>(1-\delta)^{-1}>1$. Since $\gamma>1, v$ is on the line segment joining $u$ and $w$. If $\|w\| \leqq 1$, then since $\|u\|=1$ and the unit sphere of $\mathfrak{X}$ is convex it would be necessary that $\|v\| \leqq 1$. Hence, since $\|v\|>1,\|w\|>1$. In particular, $w \notin H^{\prime}$, so if $w=\sum_{i=1}^{k} \gamma_{i} y_{i}$, some $\gamma_{i}$, say $\gamma_{j}<0$. Since $w=(1-\gamma) u+\gamma v$,

$$
\begin{aligned}
\gamma_{j} & =(1-\gamma) \beta_{j}+\gamma k^{-1}(1-\delta)^{-1} \\
& =\beta_{j}\left(1-\frac{\beta-1}{\beta-(1-\delta)^{-1}}\right)+\frac{1}{k(1-\delta)}\left(\frac{\beta-1}{\beta-(1-\delta)^{-1}}\right)<0 .
\end{aligned}
$$

Simplifying this last inequality, we find $\beta<1+k \delta \beta_{j}$. Since $\beta_{j} \leqq \beta$, we have $\beta<$ $(1-k \delta)^{-1}=(1-\varepsilon)^{-1}$ contradicting our assumption that $\beta>(1-\varepsilon)^{-1}$. Hence $\beta \leqq(1-\varepsilon)^{-1}$. Q.E.D.

7. ExAMPLES. (i) There is a reflexive, locally uniformly convex Banach space which is not $B$-convex. (This example is due to Beck.) Let $n_{i} \uparrow \infty, p_{i} \downarrow 1$. Let $\mathfrak{X}_{i}=\ell_{p_{i}}^{n_{i}}$, the space of all $n_{i}$-tuples of scalars under the norm $\left\|\left(x_{1}, \ldots, x_{n_{i}}\right)\right\|=$ $\left(\sum_{i=1}^{n_{i}}\left|x_{i}\right|^{p_{i}}\right)^{1 / p_{i}}$. Then $\mathfrak{X}_{i}$ is uniformly convex (Clarkson [3]) hence locally uniformly convex ( $\mathfrak{X}$ is locally uniformly convex if for each $x \in \mathfrak{X},\|x\|=1$, and for every $\varepsilon>0$, there exists $\delta(x, \varepsilon)>0$ such that if $y \in \mathfrak{X},\|y\|=1$, and $\|x-y\| \geqq \varepsilon$, then $\|x+y\| \leqq$ $2(1-\delta)) . \mathfrak{X}_{i}$ is finite dimensional, hence reflexive. Let $\mathfrak{X}=\sum_{i=1}^{\infty} \oplus \mathfrak{X}_{i}$, the Hilbert 
sum of the $\mathfrak{X}_{i}$. Then $x \in \mathfrak{X}$ if and only if $x=\left(x_{1}, x_{2}, \ldots\right)$ with $x_{i} \in \mathfrak{X}_{\mathfrak{i}}$ and the norm $\|x\|=\left(\sum_{i=1}^{\infty}\left\|x_{i}\right\|_{i}^{2}\right)^{1 / 2}$ is finite where $\left\|x_{i}\right\|_{i}$ is the norm of $x_{i}$ in $\mathfrak{X}_{i}$. Since the Hilbert sum of reflexive spaces is reflexive (Day [6, p. 31]) and the Hilbert sum of locally uniformly convex spaces is locally uniformly convex (Lovaglia [16]), $\mathfrak{X}$ is reflexive and locally uniformly convex. Pick $k \geqq 2, \varepsilon>0$. As $p \downarrow 1, k^{(1 / p-1)} \uparrow 1$. Choose $i$ so large that $n_{i} \geqq k$ and $k^{\left(1 / p_{i}-1\right)}>1-\varepsilon$. Notice that $\mathfrak{X}_{i}$ is imbedded isometrically in $\mathfrak{X}$. In $\mathfrak{X}_{i}$, let $x_{j}=(0, \ldots, 0,1,0, \ldots, 0)$ with the 1 in the $j$ th position. Let $y_{j} \in \mathfrak{X}$, $y_{j}=\left(0, \ldots, 0, x_{j}, 0, \ldots\right)$. Then $\left\|y_{j}\right\|=\left\|x_{j}\right\|=1$ and for $\xi_{j}= \pm 1$,

$$
\begin{aligned}
k^{-1}\left\|\sum_{j=1}^{k} \xi_{j} y_{j}\right\| & =k^{-1}\left\|\sum_{j=1}^{k} \xi_{j} x_{j}\right\|=k^{-1}\left(\sum_{j=1}^{k}\left|\xi_{j}\right|^{p_{i}}\right)^{1 / p_{i}} \\
& =k^{-1}(k)^{1 / p_{i}}=k^{1 / p_{i}-1}>1-\varepsilon .
\end{aligned}
$$

Hence $\mathfrak{X}$ is not $k, \varepsilon$-convex. Since $k$ and $\varepsilon$ were arbitrary, $\mathfrak{X}$ is not $B$-convex.

(ii) R. C. James [12] has given an example of a nonreflexive Banach space which has difficiency (codimension) 1 in its second conjugate under the natural imbedding and is isometrically isomorphic to its second conjugate. In light of Corollary II.2, if there existed a nonreflexive $B$-convex space, this would seem to be as likely a candidate as any. However, we can show that it is not $B$-convex. James discovered this independently.

$\Im$ is the set of all sequences $x=\left\{x_{n}\right\}$ of real numbers such that $\lim _{n} x_{n}=0$ and the norm $\|x\|$ is finite where $\|x\|^{2}=\sup \left[\left(x_{p_{1}}-x_{p_{n}}\right)^{2}+\sum_{i=1}^{n-1}\left(x_{p_{i+1}}-x_{p_{i}}\right)^{2}\right]$ and the sup extends over all $n \geqq 2$ and all integers $1 \leqq p_{1}<p_{2}<\cdots<p_{n}$. Without loss of generality (see Corollary II.7), we consider $\Im$ with the norm $\|\cdot\|$ which is easily shown to be equivalent to $\|\cdot\| \|$ and which is given by $\|x\|^{2}=\sup \sum_{i=1}^{n}\left(x_{p_{i}}-x_{q_{i}}\right)^{2}$ where the sup extends over all $n \geqq 1$ and all integers $1 \leqq q_{1}<p_{1} \leqq q_{2}<p_{2} \leqq \cdots \leqq$ $q_{n}<p_{n}$. If $\mathbf{p}=\left(p_{1}, \ldots, p_{n}\right)$ and $\mathbf{q}=\left(q_{1}, \ldots, q_{n}\right)$, we use the notation

$$
x^{2}(\mathbf{p}, \mathbf{q})=\sum_{i=1}^{n}\left(x_{p_{i}}-x_{q_{i}}\right)^{2}
$$

In this norm we see the character of a variation norm in that it is the difference between members of the sequence $\left\{x_{n}\right\}$ which give rise to its norm and not (for example) the size of the individual members. However, since the difference is squared, the sup is sometimes (more nearly) achieved by taking the square of one large difference rather than several small ones. Thus, $\|\{1, .4, .6,0,0, \ldots\}\|$ is $\left((1-0)^{2}\right)^{1 / 2}=1$, not $\left((1-.4)^{2}+(.4-.6)^{2}+(.6-0)^{2}\right)^{1 / 2}=\sqrt{ } .86$. By balancing one big jump against several smaller ones, we can construct elements of $\Im$ which (nearly) achieve their norms in several ways. Thus

$$
\|\{1,1 / 3,2 / 3,0,0, \ldots\}\|^{2}=(1-0)^{2}=(1-1 / 3)^{2}+(1 / 3-2 / 3)^{2}+(2 / 3-0)^{2} .
$$


By exploiting phenomena of this sort, we can pick $k \geqq 2$ and $\varepsilon>0$ and construct unit vectors $x_{1}, \ldots, x_{k} \in \mathfrak{J}$ with the property that there are $2^{k}$ sets of $\mathbf{p}$ 's and $\mathbf{q}$ 's such that each $x_{i}^{2}(\mathbf{p}, \mathbf{q}) \geqq(1-\varepsilon)^{2}$ and what is more for each choice of $\lambda_{1}= \pm 1, \ldots$, $\lambda_{k}= \pm 1$, the vectors $\lambda_{1} x_{1}, \ldots, \lambda_{k} x_{k}$ reinforce each other on some one of the p's and $\mathbf{q}$ 's so that $\left(\lambda_{1} x_{1}+\cdots+\lambda_{k} x_{k}\right)^{2}(\mathbf{p}, \mathbf{q}) \geqq k^{2}(1-\varepsilon)^{2}$, hence $\left\|\lambda_{1} x_{1}+\cdots+\lambda_{k} x_{k}\right\| \geqq$ $k(1-\varepsilon)$. The actual details of this construction involve an exceedingly tedious and cumbersome calculation which seems to add little insight, so we omit it here. Details can be found in the author's Ph.D. thesis (Giesy [10]).

II. Related spaces. In this section we shall explore conditions under which $B$ convexity is preserved between related spaces.

1. THEOREM. Let $\mathfrak{X}$ be a Banach space. $\mathfrak{X}$ is $A, k, \varepsilon$-convex if and only if $\mathfrak{X}^{* *}$ is $A, k, \varepsilon$-convex.

Proof. Since $\mathfrak{X}$ is isometrically isomorphic to a subspace of $\mathfrak{X}^{* *}$, if $\mathfrak{X}^{* *}$ is $A, k, \varepsilon$-convex, then $\mathfrak{X}$ also is (Remark I.2(c1)).

The converse depends on a theorem of Goldstine (see, e.g., Dunford and Schwartz [7, V.4.5]), which states that if $S, S^{* *}$ are the unit spheres of $\mathfrak{X}, \mathfrak{X}^{* *}$ respectively and $\kappa: \mathfrak{X} \rightarrow \mathfrak{X}^{* *}$ is the natural embedding of $\mathfrak{X}$ into $\mathfrak{X}^{* *}$, then $\kappa S$ is dense in $S^{* *}$ in the $\mathfrak{X}^{*}$-topology of $S^{* *}$.

Now choose $X_{1}, \ldots, X_{k} \in S^{* *}$. Let $\delta>0$. Pick $\zeta_{1}, \ldots, \zeta_{n} \in A$ so that for each $\zeta \in A$, there is $j(\zeta)$ such that $\left|\zeta-\zeta_{j(\xi)}\right| \leqq \delta$ (every element of $A$ is within $\delta$ of some one of the finite set $\left.\left\{\zeta_{i}\right\}\right)$. Let $\xi=\left(\xi_{1}, \ldots, \xi_{k}\right)$ be a $k$-tuple of elements from $\left\{\zeta_{1}, \ldots, \zeta_{n}\right\}$. Then $\left\|\sum_{i=1}^{k} \xi_{i} X_{i}\right\|=\sup _{\|f\|=1}\left|\left(\sum_{i=1}^{k} \xi_{i} X_{i}\right)(f)\right|$ where $f \in \mathfrak{X}^{*}$. Pick $f_{\xi} \in \mathfrak{X}^{*},\left\|f_{\xi}\right\|=1$ so that $\left|\left(\sum_{i=1}^{k} \xi_{i} X_{i}\right)\left(f_{\xi}\right)\right|>\left\|\sum_{i=1}^{k} \xi_{i} X_{i}\right\|-\delta$. For each $i$, consider the $\mathfrak{X}^{*}$-neighborhood $N$ of $X_{i}$ determined by $\delta$ and the $n^{k}$ functionals $f_{\xi}$ where $\xi$ ranges over all $k$-tuples from the set $\left\{\zeta_{1}, \ldots, \zeta_{n}\right\}$. Since $\kappa S$ is dense in $S^{* *}$, there is a vector $x_{i} \in S$ such that $\kappa x_{i} \in N$. This means that $\left|X_{i}\left(f_{\xi}\right)-\left(\kappa x_{i}\right)\left(f_{\xi}\right)\right|=\left|X_{i}\left(f_{\xi}\right)-f_{\xi}\left(x_{i}\right)\right|<\delta$ for each $\xi$. Since $\mathfrak{X}$ is $A, k, \varepsilon$-convex, there exist $\lambda_{1}, \ldots, \lambda_{k} \in A$ such that $k^{-1}\left\|\sum_{i=1}^{k} \lambda_{i} x_{i}\right\|<1-\varepsilon+\delta$. For each $\lambda_{i}$, let $j=j\left(\lambda_{i}\right), \xi_{i}=\zeta_{j}$ so that $\left|\lambda_{i}-\xi_{i}\right| \leqq \delta$ and $\xi=\left(\xi_{1}, \ldots, \xi_{k}\right)$ is a $k$-tuple of elements from $\left\{\zeta_{1}, \ldots, \zeta_{n}\right\}$. Then

$$
\begin{aligned}
k^{-1}\left\|\sum_{i=1}^{k} \xi_{i} x_{i}\right\| & \leqq k^{-1}\left\|\sum_{i=1}^{k} \lambda_{i} x_{i}\right\|+k^{-1}\left\|\sum_{i=1}^{k}\left(\lambda_{i}-\xi_{i}\right) x_{i}\right\| \\
& <1-\varepsilon+\delta+k^{-1} \sum_{i=1}^{k}\left|\lambda_{i}-\xi_{i}\right|\left\|x_{i}\right\| \\
& \leqq 1-\varepsilon+\delta+k^{-1} \sum_{i=1}^{k} \delta=1-\varepsilon+2 \delta .
\end{aligned}
$$




$$
\begin{aligned}
k^{-1}\left\|\sum_{i=1}^{k} \xi_{i} X_{i}\right\| & <k^{-1}\left|\left(\sum_{i=1}^{k} \xi_{i} X_{i}\right)\left(f_{\xi}\right)\right|+k^{-1}(k \delta) \\
& \leqq k^{-1}\left|\sum_{i=1}^{k} \xi_{i}\left(X_{i} f_{\xi}-f_{\xi} x_{i}\right)\right|+k^{-1}\left|\sum_{i=1}^{k} \xi_{i} f_{\xi}\left(x_{i}\right)\right|+\delta \\
& \leqq k^{-1} \sum_{i=1}^{k}\left|\xi_{i}\right| \cdot\left|X_{i} f_{\xi}-f_{\xi} x_{i}\right|+k^{-1}\left|f_{\xi}\left(\sum_{i=1}^{k} \xi_{i} x_{i}\right)\right|+\delta \\
& <k^{-1} \sum_{i=1}^{k} \delta+k^{-1}\left\|f_{\xi}\right\| \cdot\left\|\sum_{i=1}^{k} \xi_{i} x_{i}\right\|+\delta \\
& \leqq \delta+1-\varepsilon+2 \delta+\delta=1-\varepsilon+4 \delta .
\end{aligned}
$$

Since for every $\delta>0$ there exist $\xi_{1}, \ldots, \xi_{k} \in A$ such that $k^{-1}\left\|\sum_{i=1}^{k} \xi_{i} X_{i}\right\|<1-\varepsilon+4 \delta$, $\inf k^{-1}\left\|\sum_{i=1}^{k} \xi_{i} X_{i}\right\| \leqq 1-\varepsilon$ where the inf extends over all $k$-tuples from $A$. Since this is true for all $k$-tuples from $S^{* *}, a_{k}\left(\mathfrak{X}^{* *}, A\right) \leqq 1-\varepsilon$ so $\mathfrak{X}^{* *}$ is $A, k, \varepsilon$-convex. Q.E.D.

2. Corollary. Let $\mathfrak{X}$ be a NLS. $\mathfrak{X}$ is $A, k, \varepsilon$-convex if and only if $\mathfrak{X}^{* *}$ is $A, k, \varepsilon-$ convex.

Proof. Let $\mathfrak{Y}$ be the completion of $\mathfrak{X}$. Then vector space operations can be defined on $\mathfrak{Y}$ so that $\mathfrak{Y}$ is a Banach space and $\mathfrak{X}$ is a sub-NLS of $\mathfrak{Y}$. $\mathfrak{X}$ is dense in $\mathfrak{Y}$ so by Remark I.2(c3), $\mathfrak{Y}$ is $A, k, \varepsilon$-convex. Every element $y^{*}$ in $\mathfrak{Y} *$ defines a linear functional $x^{*}$ on $\mathfrak{X}$ by $x^{*}=y^{*} \mid \mathfrak{X}$, the restriction of $y^{*}$ to $\mathfrak{X}$, and $\left\|x^{*}\right\|=\left\|y^{*}\right\|$. Every $x^{*} \in \mathfrak{X}^{*}$ has a unique continuous linear extension $y^{*}$ to $\mathfrak{Y}$, and $\left\|x^{*}\right\|=\left\|y^{*}\right\|$. So in a natural way $\mathfrak{X}^{*}=\mathfrak{Y}^{*}$, hence $\mathfrak{X}^{* *}=\mathfrak{Y}^{* *}$. Since $\mathfrak{Y}^{* *}$ is $A, k, \varepsilon$-convex (Theorem $1), \mathfrak{X}^{* *}$ is $A, k, \varepsilon$-convex. The converse direction follows since if $\mathfrak{X}^{* *}=\mathfrak{Y}^{* *}$ is $A, k, \varepsilon$-convex, $\mathfrak{Y}$ is $A, k, \varepsilon$-convex and since $\mathfrak{X} \subset \mathfrak{Y}, \mathfrak{X}$ is $A, k, \varepsilon$-convex by Remark I.2(c1). Q.E.D.

\section{THEOREM. Let $\mathfrak{X}$ be a Banach space. $\mathfrak{X}$ is $B$-convex if and only if $\mathfrak{X}$ is B-convex.}

Proof. We will prove that if $\mathfrak{X}$ is not $B$-convex, then $\mathfrak{X}^{*}$ is not $B$-convex. The entire theorem follows from this, for, if it is true, then, if $\mathfrak{X}^{*}$ is $B$-convex, then $\mathfrak{X}$ is $B$-convex, and if $\mathfrak{X}$ is $B$-convex, $\mathfrak{X}^{* *}$ is $B$-convex by Theorem 1 , and since $\mathfrak{X}^{* *}=\left(\mathfrak{X}^{*}\right)^{*}$, this would imply $\mathfrak{X}^{*}$ is $B$-convex.

Now suppose $\mathfrak{X}$ is not $B$-convex. Choose $k \geqq 2, \varepsilon>0$. The idea of this proof is to get a good enough approximation of a finite dimensional $\ell_{1}$ in $\mathfrak{X}$ of sufficiently high dimension in order to get a good enough copy of a finite dimensional $\ell_{\infty}$ in $\mathfrak{X}^{*}$ of sufficiently high dimension to show that $\mathfrak{X}^{*}$ is not $k, \varepsilon$-convex (see Example I.3(iv)). 
Pick $\delta, 0<\delta<\varepsilon$, and let $n=2^{k}$. By Lemma I.6, there are vectors $x_{1}, \ldots, x_{n} \in S$ such that for all scalars $\alpha_{i}, 1 \leqq i \leqq n$, we have $(1-\delta) \sum_{i=1}^{n}\left|\alpha_{i}\right| \leqq\left\|\sum_{i=1}^{n} \alpha_{i} x_{i}\right\| \leqq \sum_{i=1}^{n}\left|\alpha_{i}\right|$. From this we see easily that the $x_{i}$ are linearly independent. Define linear functionals $g_{j}$ on the linear span of $x_{1}, \ldots, x_{n}, 1 \leqq j \leqq k$, reading $g_{j}\left(x_{i}\right)$ from the following table and extending by linearity.

$$
\begin{array}{cccccccc} 
& \multicolumn{1}{c}{\text { TABLE }} \\
& x_{1} & x_{2} & x_{3} & x_{4} & \cdots & x_{n-1} & x_{n} \\
g_{1} & +1 & -1 & +1 & -1 & \cdots & +1 & -1 \\
g_{2} & +1 & +1 & -1 & -1 & \cdots & -1 & -1 \\
\vdots & \vdots & \vdots & \vdots & \vdots & & \vdots & \vdots \\
g_{k} & +1 & +1 & +1 & +1 & \cdots & -1 & -1
\end{array}
$$

The $i$ th line consists of alternate blocks of +1 's and -1 's, each block being $2^{i-1}$ long. Notice that every sequence of $k+1$ 's and -1 's appears as a column in this table.

If $\left\|\sum_{i=1}^{n} \alpha_{i} x_{i}\right\|=1,(1-\delta) \sum_{i=1}^{n}\left|\alpha_{i}\right| \leqq 1$, so for each $j$,

$$
\begin{aligned}
(1-\delta)\left|g_{j}\left(\sum_{i=1}^{n} \alpha_{i} x_{i}\right)\right| & =(1-\delta)\left|\sum_{i=1}^{n} \alpha_{i} g_{j}\left(x_{i}\right)\right| \\
& \leqq(1-\delta) \sum_{i=1}^{n}\left|\alpha_{i}\right|\left|g_{j}\left(x_{i}\right)\right| \\
& =(1-\delta) \sum_{i=1}^{n}\left|\alpha_{i}\right| \leqq 1
\end{aligned}
$$

since $\left|g_{j}\left(x_{i}\right)\right|=1$ for all $i, j$. This tells us that $\left\|(1-\delta) g_{j}\right\| \leqq 1$ where $g_{j}$ is considered as a functional on $\operatorname{span}\left\{x_{1}, \ldots, x_{n}\right\}$. Let $f_{j}$ be a norm-preserving extension of $(1-\delta) g_{j}$ to all of $\mathfrak{X}$. Then $f_{j} \in \mathfrak{X}^{*}$ and $\left\|f_{j}\right\| \leqq 1$. Let $\xi=\left(\xi_{1}, \ldots, \xi_{k}\right)$ be a sequence of +1 's and -1 's. Pick $i, 1 \leqq i \leqq n$, so that the $i$ th column of the table defining the $g_{j}$ 's is the sequence $\xi$, that is $g_{j}\left(x_{i}\right)=\xi_{j}, 1 \leqq j \leqq k$.

$$
\begin{aligned}
k^{-1}\left\|\sum_{j=1}^{k} \xi_{j} f_{j}\right\| & \geqq k^{-1}\left|\left(\sum_{j=1}^{k} \xi_{j} f_{j}\right)\left(x_{i}\right)\right| \\
& =k^{-1}\left|\left(\sum_{j=1}^{k} \xi_{j}(1-\delta) g_{j}\right)\left(x_{i}\right)\right| \\
& =k^{-1}(1-\delta)\left|\sum_{j=1}^{k} \xi_{j} g_{j}\left(x_{i}\right)\right| \\
& =k^{-1}(1-\delta)\left|\sum_{j=1}^{k} \xi_{j}^{2}\right| \\
& =(1-\delta)>1-\varepsilon .
\end{aligned}
$$


Since $\xi$ was arbitrary, we see that $\mathfrak{X}^{*}$ is not $k$, $\varepsilon$-convex. Since $k$ and $\varepsilon$ were arbitrary $\mathfrak{X}^{*}$ is not $B$-convex. Q.E.D.

4. Corollary. Let $\mathfrak{X}$ be a NLS. $\mathfrak{X}$ is $B$-convex if and only if $\mathfrak{X}^{*}$ is.

Proof. As in the proof of Corollary 2, let $\mathfrak{Y}$ be the completion of $\mathfrak{X}$, so $\mathfrak{X}$ is dense in $\mathfrak{Y}$, and $\mathfrak{Y}$ is a Banach space. $\mathfrak{X}$ is $B$-convex if and only if $\mathfrak{Y}$ is $B$-convex if and only if $\mathfrak{Y}^{*}$ is $B$-convex if and only if $\mathfrak{X}^{*}\left(=\mathfrak{Y}^{*}\right)$ is $B$-convex. Q.E.D.

5. THEOREM. Let $\mathfrak{X}$ be a NLS, $T$ a continuous linear open transformation on $\mathfrak{X}$. If $\mathfrak{X}$ is $B$-convex, then $T(\mathfrak{X})$ is $B$-convex.

Proof. Write $\mathfrak{Y}=T(\mathfrak{X})$. Then $\mathfrak{Y}$ is a NLS. Since $T$ is continuous and linear, $T$ is bounded; i.e., there exists $M>0$ such that if $x \in \mathfrak{X},\|x\| \leqq 1$, then $\|T(x)\| \leqq M$. Since $T$ is open $T(S)$ is a neighborhood of 0 in $\mathfrak{Y}$, hence there exists $\delta>0$ such that $\{y \in \mathfrak{Y}:\|y\| \leqq \delta\} \subset T(S)$.

Now, using the $B$-convexity of $\mathfrak{X}$ choose $k$ so large that $a_{k}(\mathfrak{X}, B) \leqq \frac{1}{2} \delta M^{-1}$. Pick $y_{1}, \ldots, y_{k}$ in the unit ball of $\mathfrak{Y}$. Since $\delta y_{i} \in T(S)$, there exists $x_{i} \in S$ so that $T\left(x_{i}\right)=\delta y_{i}$. Let $\xi_{i}= \pm 1,1 \leqq i \leqq k$, so that

$$
k^{-1}\left\|\sum_{i=1}^{k} \xi_{i} x_{i}\right\| \leqq \frac{1}{2} M^{-1} \delta
$$

Then

$$
\begin{aligned}
k^{-1}\left\|\sum_{i=1}^{k} \xi_{i} y_{i}\right\| & =\delta^{-1} k^{-1}\left\|\sum_{i=1}^{k} \xi_{i} \delta y_{i}\right\| \\
& =\delta^{-1} k^{-1}\left\|\sum_{i=1}^{k} \xi_{i} T\left(x_{i}\right)\right\| \\
& =\delta^{-1} k^{-1}\left\|T\left(\sum_{i=1}^{k} \xi_{i} x_{i}\right)\right\| \\
& \leqq \delta^{-1} M k^{-1}\left\|\sum_{i=1}^{k} \xi_{i} x_{i}\right\| \\
& \leqq \delta^{-1} M\left(\frac{1}{2} M^{-1} \delta\right)=\frac{1}{2} .
\end{aligned}
$$

Thus $\mathfrak{Y}$ is $k, \frac{1}{2}$-convex, hence $B$-convex. Q.E.D.

Remarks. The condition that $T$ be open cannot be dropped. Define $T: \ell_{2} \rightarrow \ell_{1}$ by $T\left(x_{1}, x_{2}, \ldots\right)=\left(x_{1}, \frac{1}{2} x_{2}, \ldots, n^{-1} x_{n}, \ldots\right)$. Then $T$ is linear, $\|T\| \leqq 1+\pi / \sqrt{ } 6$ so $T$ is continuous, and $T\left(\ell_{2}\right)$ is dense in $\ell_{1} . \ell_{2}$ is $B$-convex (in fact, uniformly convex) and since $\ell_{1}$ is not $B$-convex, no dense subspace of $\ell_{1}$ can be $B$-convex. 
The converse of this theorem is false. Let $T: \mathfrak{X} \oplus \mathfrak{Y} \rightarrow \mathfrak{X}$ be the projection of $\mathfrak{X} \oplus \mathfrak{Y}$ onto $\mathfrak{X}$, where $\mathfrak{X}$ is $B$-convex, $\mathfrak{Y}$ is not, and $\mathfrak{X} \oplus \mathfrak{Y}$ is made into a NLS by any convenient norm, e.g., $\|(x, y)\|=\|x\|_{\mathfrak{X}}+\|y\|_{\mathfrak{\vartheta}}$. Then $T$ is continuous, linear, and open, and $T(\mathfrak{X} \oplus \mathfrak{Y})$ is $B$-convex. However, $\mathfrak{Y} \equiv 0 \oplus \mathfrak{Y}$ is not $B$-convex, hence $\mathfrak{X} \oplus \mathfrak{Y}$ is not $B$-convex. Note that kernel $(T)=0 \oplus \mathfrak{Y}$. We will prove (Corollary 10) that if $T$ is continuous, linear, and open, $\mathfrak{X}$ is $B$-convex if and only if $T(\mathfrak{X})$ and kernel $(T)$ are both $B$-convex.

6. CoROllaRY. If $\mathfrak{X}$ and $\mathfrak{Y}$ are isomorphic NLS's, $\mathfrak{X}$ is B-convex if and only if $\mathfrak{Y}$ is B-convex.

Proof. $\mathfrak{X}$ and $\mathfrak{Y}$ are isomorphic if there is a transformation $T$ on $\mathfrak{X}$ onto $\mathfrak{Y}$ which is a vector space isomorphism and a topological homeomorphism. This implies $T$ is continuous, linear, and open. Thus, if $\mathfrak{X}$ is $B$-convex, $\mathfrak{Y}$ is $B$-convex. Isomorphism is an equivalence relation on NLS's so the proof is complete.

\section{COROllary. B-convexity is invariant under equivalent renorming.}

Proof. Two norms $\|\cdot\|_{1}$ and $\|\cdot\|_{2}$ on $\mathfrak{X}$ are equivalent if $\left(\mathfrak{X},\|\cdot\|_{1}\right)$ is isomorphic to $\left(\mathfrak{X},\|\cdot\|_{2}\right)$ under the identity map. Hence, $\left(\mathfrak{X},\|\cdot\|_{1}\right)$ is $B$-convex if and only if $\left(\mathfrak{X},\|\cdot\|_{2}\right)$ is $B$-convex by Corollary 6 .

ReMARK. This result also follows directly from Beck's theorem since both the hypotheses and the conclusions of the strong law of large numbers are invariant under equivalent renorming.

8. Corollary. If $\mathfrak{X}$ and $\mathfrak{Y}$ are Banach spaces, $T$ is a continuous linear transformation on $\mathfrak{X}$ onto $\mathfrak{Y}$ and $\mathfrak{X}$ is $B$-convex, then $\mathfrak{Y}$ is $B$-convex.

Proof. Under the hypothesis, $T$ is open by the Open Mapping Theorem. Hence Theorem 5 applies.

9. THEOREM. Let $\mathfrak{X}$ be a NLS, 3 a closed subspace. $\mathfrak{X}$ is $B$-convex if and only if 3 and $\mathfrak{X} / 3$ are $B$-convex.

Proof. $\mathfrak{X} / \mathbb{B}$ is the linear space formed by taking the quotient of the linear space

$\mathfrak{X}$ by its subspace 8 . The norm in $\mathfrak{X} / 3$ is given by $\|x+3\|=\inf _{z \in \mathcal{B}}\|x+z\|$. The natural map $T: \mathfrak{X} \rightarrow \mathfrak{X} / 3$ given by $T(x)=x+3$ is continuous, linear, and open. Thus, if $\mathfrak{X}$ is $B$-convex, then $\mathbb{B}$ is $B$-convex (Remark I.2(c1)) and $\mathfrak{X} / \mathcal{B}$ is $B$-convex (Theorem 5).

Now suppose 3 and $\mathfrak{X} / 3$ are $B$-convex. By Lemma I.4, choose $m$ and $n$ so that $\mathfrak{X} / 3$ is $m, 3 / 4$-convex and $\mathbb{B}$ is $n, 5 / 6$-convex. Let $k=m n$. Pick $x_{1}, \ldots, x_{k} \in S$. Then 
$x_{1}+3, \ldots, x_{k}+8$ are in the unit ball of $\mathfrak{X} / 3$. We group these $k=m n$ vectors in $\mathfrak{X} / 8$ into $n$ groups of $m$ vectors each, and find coefficients \pm 1 to make the sum of each group small. Thus, there are $\zeta_{i}= \pm 1,1 \leqq i \leqq k$, so that letting

$$
\begin{gathered}
S_{j}=\{m(j-1)+1, \ldots, m j\} \\
\left\|\sum_{i \in S_{j}} \zeta_{i}\left(x_{i}+3\right)\right\|=\left\|\left(\sum_{i \in S_{j}} \zeta_{i} x_{i}\right)+3\right\| \leqq(1 / 4) m \quad \text { for } 1 \leqq j \leqq n .
\end{gathered}
$$

By the definition of the norm in $\mathfrak{X} / \mathcal{Z}$, we may choose $z_{j} \in \mathbb{Z}, 1 \leqq j \leqq n$, so that

$$
\left\|\left(\sum_{i \in S_{j}} \zeta_{i} x_{i}\right)+z_{j}\right\| \leqq\left\|\left(\sum_{i \in S_{j}} \zeta_{i} x_{i}\right)+3\right\|+(1 / 4) m \leqq(1 / 2) m
$$

Then

$$
\left\|z_{j}\right\| \leqq\left\|\sum_{i \in S_{j}} \zeta_{i} x_{i}\right\|+\left\|\left(\sum_{i \in S_{j}} \zeta_{i} x_{i}\right)+z_{j}\right\| \leqq m+(1 / 2) m=(3 / 2) m
$$

Using the $n, 5 / 6$-convexity of 3 , we may find $\xi_{j}= \pm 1,1 \leqq j \leqq n$, so that

$$
\left\|\sum_{j=1}^{n} \xi_{j} z_{j}\right\| \leqq(1 / 6) n(3 / 2) m=(1 / 4) k
$$

For $1 \leqq j \leqq n$, let $\lambda_{i}=\zeta_{i} \xi_{j}$ for $i \in S_{j}$. Then

$$
\begin{aligned}
\left\|\sum_{i=1}^{k} \lambda_{i} x_{i}\right\| & =\left\|\sum_{j=1}^{n} \sum_{i \in S_{j}} \xi_{j} \zeta_{i} x_{i}\right\| \\
& \leqq\left\|\sum_{i=1}^{n} \xi_{j}\left(\sum_{i \in S_{j}} \zeta_{i} x_{i}+z_{j}\right)\right\|+\left\|\sum_{j=1}^{n} \xi_{j} z_{j}\right\| \\
& \leqq \sum_{j=1}^{n}\left\|\sum_{i \in S_{j}} \zeta_{i} x_{i}+z_{j}\right\|+(1 / 4) k \\
& \leqq \sum_{j=1}^{n}(1 / 2) m+(1 / 4) k \\
& =(1 / 2) m n+(1 / 4) k=(3 / 4) k .
\end{aligned}
$$

Thus $\mathfrak{X}$ is $k, 1 / 4$ convex, hence $B$-convex. Q.E.D.

10. Corollary. Let $\mathfrak{X}$ be a NLS and $T$ a continuous linear open transformation on $\mathfrak{X}$ with kernel 3 . Then $\mathfrak{X}$ is $B$-convex if and only if both $\mathbb{Z}$ and $T(\mathfrak{X})$ are $B$-convex. 
Proof. If $\mathfrak{X}$ is $B$-convex, since 8 is a subspace of $\mathfrak{X}, \mathbb{B}$ is $B$-convex (Remark I.2(c1)) and by the hypotheses on $T$ and Theorem $5, T(\mathfrak{X})$ is $B$-convex.

Suppose 8 and $T(\mathfrak{X})$ are both $B$-convex. Since $T$ is continuous, 8 is closed in $\mathfrak{X}$. Define $U: T(\mathfrak{X}) \rightarrow \mathfrak{X} / 3$ by $U(T(x))=x+8$.

It is easily verified that $U$ is a well-defined, continuous, linear, open transformation from $T(\mathfrak{X})$ onto $\mathfrak{X} / \mathbb{Z}$, and since $T(\mathfrak{X})$ is $B$-convex, $\mathfrak{X} / \mathbb{Z}$ is $B$-convex (Theorem 5). Since $\mathbb{Z}$ is also $B$-convex, $\mathfrak{X}$ is $B$-convex (Theorem 9 ).

11. Lemma. Let $\mathfrak{X}_{1}, \ldots, \mathfrak{X}_{n}$ be $N L S$ 's, and let $\mathfrak{Y}=\mathfrak{X}_{1} \oplus \cdots \oplus \mathfrak{X}_{n}$, the direct sum of the $\mathfrak{X}_{i}$ under component-wise arithmetic and norm $\|y\|_{\mathfrak{Y}}=\left\|x_{1}\right\|_{\mathfrak{X}_{1}}+\cdots+\left\|x_{n}\right\|_{\mathfrak{X}_{n}}$ for $y \in \mathfrak{Y}, y=\left(x_{1}, \ldots, x_{n}\right)$. Then $\mathfrak{Y}$ is $B$-convex if and only if all of $\mathfrak{X}_{1}, \ldots, \mathfrak{X}_{n}$ are.

Proof. First each $\mathfrak{X}_{i}$ is isometrically isomorphic in the natural way to the subspace $0 \oplus \cdots \oplus 0 \oplus \mathfrak{X}_{i} \oplus 0 \oplus \cdots \oplus 0$ of $\mathfrak{Y}$, so if $\mathfrak{Y}$ is $B$-convex, each $\mathfrak{X}_{i}$ is.

For the converse, we proceed by induction on $n$. For $n=1$, the result is trivial. Suppose the theorem is true for $n-1$. Suppose $\mathfrak{X}_{1}, \ldots, \mathfrak{X}_{n}$ are $B$-convex. Define $T: \mathfrak{Y} \rightarrow \mathfrak{X}_{n}$ by $T\left(x_{1}, \ldots, x_{n}\right)=x_{n}$. $T$ is easily seen to be continuous, linear, and open with $B$-convex image $\mathfrak{X}_{n}$ and with kernel $\mathfrak{X}_{1} \oplus \cdots \oplus \mathfrak{X}_{n-1} \oplus 0$ which is isometrically isomorphic to $\mathfrak{X}_{1} \oplus \cdots \oplus \mathfrak{X}_{n-1}$ which is $B$-convex by the hypotheses of induction. Hence by Corollary $10, \mathfrak{Y}$ is $B$-convex. Q.E.D.

12. THEOREM. Let $\mathfrak{X}$ be a Banach space and $\mathfrak{X}_{1}, \ldots, \mathfrak{X}_{n}$ linear subspaces of $\mathfrak{X}$ such that $\mathfrak{X}$ is the linear span of $\mathfrak{X}_{1}, \ldots, \mathfrak{X}_{n}$. Then $\mathfrak{X}$ is $B$-convex if and only if $\mathfrak{X}_{1}, \ldots, \mathfrak{X}_{n}$ all are.

Proof. As usual, by Remark I.2(c1), if $\mathfrak{X}$ is $B$-convex, each $\mathfrak{X}_{i}$ is also.

For the converse, suppose $\mathfrak{X}_{1}, \ldots, \mathfrak{X}_{n}$ are $B$-convex and closed in $\mathfrak{X}$. Then each $\mathfrak{X}_{i}$ is complete. Let $\mathfrak{Y}=\mathfrak{X}_{1} \oplus \cdots \oplus \mathfrak{X}_{n}$ as in Lemma 11 . Then by that lemma, $\mathfrak{Y}$ is $B$-convex and $\mathfrak{Y}$ is easily seen to be complete. Let $T: \mathfrak{Y} \rightarrow \mathfrak{X}$ be defined by $T\left(x_{1}, \ldots, x_{n}\right)=x_{1}+\cdots+x_{n} . T$ is clearly linear

$$
\left\|T\left(x_{1}, \ldots, x_{n}\right)\right\|_{\mathfrak{X}}=\left\|x_{1}+\cdots+x_{n}\right\| \leqq\left\|x_{1}\right\|+\cdots+\left\|x_{n}\right\|=\left\|\left(x_{1}, \ldots, x_{n}\right)\right\|_{\mathfrak{Y}}
$$

so $\|T\| \leqq 1$, and hence $T$ is continuous. Since $\mathfrak{X}$ is the linear span of $\mathfrak{X}_{1}, \ldots, \mathfrak{X}_{n}$, $T$ is onto. Hence by Corollay $8, \mathfrak{X}$ is $B$-convex.

Now if each $\mathfrak{X}_{\mathfrak{i}}$ is $B$-convex but not necessarily closed, by Remark I.2(c3) cl $\mathfrak{X}_{i}$ is $B$-convex, and the hypothesis of this theorem is satisfied with each $\mathfrak{X}_{i}$ replaced by $\mathrm{cl} \mathfrak{X}_{i}$ and the previous argument shows that $\mathfrak{X}$ is $B$-convex.

REMARK. We cannot weaken the hypotheses to state " $\mathfrak{X}$ is a NLS." Let $\mathfrak{\Im}$ be James' space (see Example I.7(ii)). Define $\mathfrak{S}_{1}=\left\{\left(x_{1}, 0, x_{3}, 0, \ldots\right) \in \mathfrak{\Im}\right\}, \mathfrak{S}_{2}=$ 
$\left\{\left(0, x_{2}, 0, x_{4}, \ldots\right) \in \mathfrak{\Im}\right\}$. Then $\mathfrak{S}_{1}$ and $\mathfrak{S}_{2}$ are isomorphic to Hilbert space and $\operatorname{sp}\left(\mathfrak{S}_{1}, \mathfrak{S}_{2}\right)$ is dense in $\mathfrak{\Im}$ (James [11]). By Example I.3(ii) and Corollary 6, $\mathfrak{S}_{1}$ and $\mathfrak{S}_{2}$ are $B$-convex. However, since $\mathfrak{\Im}$ is not $B$-convex $\operatorname{sp}\left(\mathfrak{S}_{1}, \mathfrak{S}_{2}\right)$, a NLS, is not $B$-convex by Remark I.2(c3).

13. If $\mathfrak{X}$ is a NLS of sequences, i.e., scalar-valued functions, on an index set $\mathscr{T}$ and for each $t \in \mathscr{T}, \mathfrak{X}_{t}$ is a NLS, we may form a new NLS, $P_{\mathfrak{X}} \mathfrak{X}_{t}$, the set of all sequences $\{x(t)\}$ with $x(t) \in \mathfrak{X}_{t}$ such that the sequence of scalars $\{\|x(t)\|\}$ is in $\mathfrak{X}$. It is customary to impose some restrictions on $\mathfrak{X}$ which we shall give presently in order to make the situation manageable. Such a restricted $\mathfrak{X}$ will be called a proper function space. M. M. Day showed ([4] and [5]) that if $\mathfrak{X}$ is a uniformly convex proper function space and the $\mathfrak{X}_{t}$ are uniformly convex with a common modulus of convexity (the function $\delta(\cdot)$ associated with a uniformly convex space is called its modulus of convexity; see Example I.3(ii)), then $P_{\mathfrak{X}} \mathfrak{X}_{t}$ is uniformly convex. Following Day's techniques, we show that if $\mathfrak{X}$ is uniformly convex and $\mathfrak{X}_{t}$ is $k, \varepsilon$-convex for each $t \in \mathscr{T}$, then $P_{\mathfrak{X}} \mathfrak{X}_{t}$ is $k, \delta$-convex for suitable $\delta$ (Theorem 17).

14. Definitions. Let $\mathscr{T}$ be an index set and $\mathfrak{X}$ a NLS of real-valued functions on $\mathscr{T}$. For each $t \in \mathscr{T}$, let $\mathfrak{X}_{t}$ be a NLS (all $\mathfrak{X}_{t}$ over the same scalar field). Denote by $P_{\mathfrak{X}} \mathfrak{X}_{t}$ the set of all functions $x$ on $\mathscr{T}$ such that $x(t) \in \mathfrak{X}_{t}$ and if the real-valued function $\xi$ on $\mathscr{T}$ is defined by $\xi(t)=\|x(t)\|, \xi \in \mathfrak{X}$. Norm $P_{\mathfrak{X}} \mathfrak{X}_{t}$ by $\|x\|=\|\xi\|$ with $x$ and $\xi$ as above.

Call a NLS $\mathfrak{X}$ of real-valued functions on $\mathscr{T}$ a proper space of functions if $\mathfrak{X}$ satisfies the condition that whenever $\xi \in \mathfrak{X}$ and $\zeta$ is a real-valued function on $\mathscr{T}$ with $|\zeta(t)| \leqq|\xi(t)|$ for each $t \in \mathscr{T}$, then $\zeta \in \mathfrak{X}$ and $\|\zeta\| \leqq\|\xi\|$.

It is known (Day [6, p. 31]) that if $\mathfrak{X}$ is a proper space of functions on $\mathscr{T}$, then $P_{\mathfrak{X}} \mathfrak{X}_{t}$ is a NLS, and is complete if $\mathfrak{X}$ and all $\mathfrak{X}_{t}$ are complete.

15. Lemma. Let $\mathfrak{X}$ be a uniformly convex NLS. Then there exists a function $\delta^{\prime}(\cdot)$ with $\delta^{\prime}(\varepsilon)>0$ for $\varepsilon>0$ such that if $x, y \in S$ and $\|x-y\| \geqq \varepsilon$, then $\|x+y\| \leqq$ $2\left(1-\delta^{\prime}(\varepsilon)\right)$.

(This lemma is stated by Day in [5, Lemma 1]. The proof given here is due to this author.)

Proof. Let $\delta(\cdot)$ be the function given by the uniform convexity of $\mathfrak{X}$ and let $\delta^{\prime}(\varepsilon)=\min ((1 / 3) \delta(\varepsilon / 3), \varepsilon / 6,1 / 4)$. Then for $\varepsilon>0, \delta^{\prime}(\varepsilon)>0$.

Now let $x, y \in S$ and suppose $\|x+y\|>2\left(1-\delta^{\prime}(\varepsilon)\right)$. Now

$$
\|x\| \geqq\|x+y\|-\|y\|>2\left(1-\delta^{\prime}(\varepsilon)\right)-1=1-2 \delta^{\prime}(\varepsilon)>0
$$

since $\delta^{\prime}(\varepsilon) \leqq 1 / 4$, so $x \neq 0$. Similarly $y \neq 0$. Let $\lambda=\|x\|^{-1}, \mu=\|y\|^{-1}$. Then $\|\lambda x\|=$ $\|\mu y\|=1$. Also,

$$
\begin{aligned}
\|(1-\lambda) x\|=|1-\lambda| \cdot\|x\| & =\left|1-\|x\|^{-1}\right| \cdot\|x\| \\
& =|\|x\|-1|<2 \delta^{\prime}(\varepsilon)
\end{aligned}
$$


since $1 \geqq\|x\|>1-2 \delta^{\prime}(\varepsilon)$. Similarly $\|(1-\mu) y\|<2 \delta^{\prime}(\varepsilon)$. Therefore

$$
\begin{aligned}
\|\lambda x+\mu y\| & \geqq\|x+y\|-\|(1-\lambda) x\|-\|(1-\mu) y\| \\
& >2\left(1-\delta^{\prime}(\varepsilon)\right)-2 \delta^{\prime}(\varepsilon)-2 \delta^{\prime}(\varepsilon) \\
& =2\left(1-3 \delta^{\prime}(\varepsilon)\right) \geqq 2(1-\delta(\varepsilon / 3)) .
\end{aligned}
$$

Therefore, since $\lambda x$ and $\mu y$ are unit vectors, $\|\lambda x-\mu y\|<\varepsilon / 3$. Hence

$$
\begin{aligned}
\|x-y\| & \leqq\|\lambda x-\mu y\|+\|(1-\lambda) x\|+\|(1-\mu) y\| \\
& <\varepsilon / 3+2 \delta^{\prime}(\varepsilon)+2 \delta^{\prime}(\varepsilon) \leqq \varepsilon .
\end{aligned}
$$

Hence, if $x, y \in S$ with $\|x-y\| \geqq \varepsilon$, we must have

$$
\|x+y\| \leqq 2\left(1-\delta^{\prime}(\varepsilon)\right) \text {. Q.E.D. }
$$

16. LEMMA. Let $\mathfrak{X}$ be a uniformly convex proper space of functions on $\mathscr{T}$, and let $n$ be a positive integer and $\varepsilon>0$. Then there exists $\delta=\delta(\mathfrak{X}, n, \varepsilon)>0$ such that if $\mathscr{T}_{1}, \ldots, \mathscr{T}_{n}$ are pairwise disjoint with $\mathscr{T}=\bigcup \mathscr{T}_{i}$, and if $\xi \in \mathfrak{X}$ with $\|\xi\| \leqq 1$, and if for each $i, \xi_{i} \in \mathfrak{X}$ with $\left|\xi_{i}(t)\right| \leqq|\xi(t)|$ for $t \notin \mathscr{T}_{i}$ and $\left|\xi_{i}(t)\right| \leqq|(1-\varepsilon) \xi(t)|$ for $t \in \mathscr{T}_{i}$, then for some $i, 1 \leqq i \leqq n,\left\|\xi_{i}\right\| \leqq 1-\delta$.

Proof. We will make heavy use of the "monotonicity" property which characterizes proper spaces of functions. If $\|\xi\|=0$, the proof is clear, so we shall assume $\|\xi\|>0$. Define $\phi \in \mathfrak{X}$ by $\phi(t)=|\xi(t)| /\|\xi\|$. Then $\phi(t) \geqq 0$ and $\|\phi\|=1$. Let $\chi_{i}$ be the characteristic function of $\mathscr{T}_{i}$ so $\chi_{i}(t)=0$ resp. 1 if $t \notin \mathscr{T}_{i}$ resp. $t \in \mathscr{T}_{i}$. Define $\phi_{i}(t)=$ $\left(1-\chi_{i}(t) \varepsilon\right) \phi(t)$. Then $\phi_{i}(t) \geqq 0$ and $\left|\xi_{i}(t)\right| \leqq \phi_{i}(t)$, so $\left\|\xi_{i}\right\| \leqq\left\|\phi_{i}\right\|$. We will find a $\delta>0$ depending only on $\mathfrak{X}, n$, and $\varepsilon$ such that for some $i,\left\|\phi_{i}\right\| \leqq 1-\delta$, hence $\left\|\xi_{i}\right\| \leqq 1-\delta$. First suppose $\varepsilon \leqq 2 / 3$.

Let $\eta_{i}(t)=\varepsilon \phi(t) \chi_{i}(t)$ and $\eta(t)=\varepsilon \phi(t)$. Then since $\left\{\mathscr{T}_{1}, \ldots, \mathscr{T}_{n}\right\}$ is a partition of $\mathscr{T}, \sum_{i=1}^{n} \eta_{i}=\eta=\varepsilon \phi$, so $\varepsilon=\|\varepsilon \phi\|=\|\eta\|=\left\|\sum_{i=1}^{n} \eta_{i}\right\| \leqq \sum_{i=1}^{n}\left\|\eta_{i}\right\|$ so, for some $i, 1 \leqq i \leqq n$, $\left\|\eta_{i}\right\| \geqq \varepsilon / n$. Fix this $i$ for the remainder of this proof.

Define $\zeta(t)=\left(1-\chi_{i}(t)(\varepsilon / 2)\right) \phi(t), \zeta^{\prime}(t)=\left(1-\chi_{i}(t)(3 \varepsilon / 2)\right) \phi(t)$. Then since $\varepsilon \leqq 2 / 3$, for each $t, 0 \leqq \zeta^{\prime}(t) \leqq \zeta(t) \leqq \phi(t)$ so $\left\|\zeta^{\prime}\right\| \leqq\|\zeta\| \leqq 1$. Now

$$
\begin{aligned}
\zeta(t)-\zeta^{\prime}(t) & =\left(1-\chi_{i}(t)(\varepsilon / 2)\right) \phi(t)-\left(1-\chi_{i}(t)(3 \varepsilon / 2)\right) \phi(t) \\
& =\varepsilon \chi_{i}(t) \phi(t)=\eta_{i}(t)
\end{aligned}
$$

so $\zeta-\zeta^{\prime}=\eta_{i}$

$$
\begin{aligned}
\zeta(t)+\zeta^{\prime}(t) & =\left(1-\chi_{i}(t)(\varepsilon / 2)\right) \phi(t)+\left(1-\chi_{i}(t)(3 \varepsilon / 2)\right) \phi(t) \\
& =2\left(1-\chi_{i}(t) \varepsilon\right) \phi(t)=2 \phi_{i}(t)
\end{aligned}
$$


So $\zeta+\zeta^{\prime}=2 \phi_{i}$. Thus $\left\|\zeta-\zeta^{\prime}\right\|=\left\|\eta_{i}\right\| \geqq \varepsilon / n$, so if $\delta^{\prime}(\cdot)$ is the function in $\mathfrak{X}$ given by Lemma 15, $\left\|\zeta+\zeta^{\prime}\right\| \leqq 2\left(1-\delta^{\prime}(\varepsilon / n)\right)$. Thus letting $\delta=\delta^{\prime}(\varepsilon / n),\left\|\phi_{i}\right\| \leqq 1-\delta$.

Now if $\varepsilon>2 / 3$, the hypotheses of the lemma are satisfied with $\varepsilon$ replaced by $\varepsilon^{\prime}=2 / 3 . \delta\left(\mathfrak{X}, n, \varepsilon^{\prime}\right)$ given by the above proof is a suitable choice for $\delta(\mathfrak{X}, n, \varepsilon)$. Q.E.D.

17. THEOREM. Let $\mathfrak{X}$ be a uniformly convex proper space of functions on an index set $\mathscr{T}$, and for each $t \in \mathscr{T}$, let $\mathfrak{X}_{t}$ be a $k, \varepsilon$-convex space. Then there exists $\delta>0$ such that $P_{\mathfrak{X}} \mathfrak{X}_{t}$ is $k, \delta$-convex.

Proof. The proof divides naturally into two parts, the first a special case and the second the general case. For brevity, we write $\mathfrak{Y}=P_{\mathfrak{X}} \mathfrak{X}_{t}$.

Part I. Let $\delta_{1}=\delta(\mathfrak{X}, n, \varepsilon)$ of Lemma 16 with $n=2^{k}$. Let $\left.x_{1}, \ldots, x_{k} \in \mathfrak{Y}\right),\left\|x_{i}\right\| \leqq 1$ with the additional restriction that $\left\|x_{i}(t)\right\|=\left\|x_{1}(t)\right\|$ for $2 \leqq i \leqq k$. Denote this common value $\left\|x_{1}(t)\right\|$ by $\xi(t)$. Using the $k, \varepsilon$-convexity of each $\mathfrak{X}_{t}$, we partition $\mathscr{T}$ into $2^{k}$ sets $\left\{\mathscr{T}_{\lambda}: \lambda=\left(\lambda_{1}, \ldots, \lambda_{k}\right), \lambda_{i}= \pm 1,1 \leqq i \leqq k\right\}$ with the property that if $t \in \mathscr{T}_{\lambda}$, then $k^{-1}\left\|\sum_{i=1}^{k} \lambda_{i} x_{i}(t)\right\| \leqq(1-\varepsilon) \xi(t)$. Define $\xi_{\lambda}$ by $\xi_{\lambda}(t)=k^{-1}\left\|\sum_{i=1}^{k} \lambda_{i} x_{i}(t)\right\|$. Then by the triangle inequality, $0 \leqq \xi_{\lambda}(t) \leqq \xi(t)$ and for $t \in \mathscr{T}_{\lambda}, 0 \leqq \xi_{\lambda}(t) \leqq(1-\varepsilon) \xi(t)$, so by Lemma 16, there is a $\lambda$ such that $\left\|\xi_{\lambda}\right\| \leqq 1-\delta_{1}$. But by the definition of norm in $\mathfrak{Y},\left\|\xi_{\lambda}\right\|=k^{-1}\left\|\sum_{i=1}^{k} \lambda_{i} x_{i}\right\|$. Hence, given $k$ vectors $x_{1}, \ldots, x_{k}$ in $\mathfrak{Y},\left\|x_{i}\right\| \leqq 1$, such that $\left\|x_{i}(t)\right\|=\left\|x_{1}(t)\right\|, 2 \leqq i \leqq k, t \in \mathscr{T}$, there exist $\lambda_{i}= \pm 1,1 \leqq i \leqq k$ such that $k^{-1}\left\|\sum_{i=1}^{k} \lambda_{i} x_{i}\right\| \leqq 1-\delta_{1}$.

Part II. Pick $\delta_{2}$ so that $\delta_{1}>\delta_{2}>0$. Let $\delta^{\prime}(\cdot)$ be the function in $\mathfrak{X}$ given by Lemma 15 , and define $\delta_{3}$ by requiring $\delta^{\prime}\left(\delta_{2}\right)=\frac{1}{2} k \delta_{3}$. Then $\delta_{3}>0$. Let $\delta=\min \left(\delta_{1}-\delta_{2}, \delta_{3}\right)$. Then $\delta>0$. Let $x_{1}, \ldots, x_{k} \in \mathfrak{Y},\left\|x_{i}\right\| \leqq 1$ for $1 \leqq i \leqq k$. Let $\xi_{i}(t)=\left\|x_{i}(t)\right\|$, so $\xi_{i} \in \mathfrak{X}$ and $\left\|\xi_{i}\right\|=\left\|x_{i}\right\| \leqq 1$. $\left\|\sum_{i=1}^{k} x_{i}(t)\right\| \leqq \sum_{i=1}^{k}\left\|x_{i}(t)\right\|=\sum_{i=1}^{k} \xi_{i}(t)$, so by a componentwise comparison, $\left\|\sum_{i=1}^{k} x_{i}\right\| \leqq\left\|\sum_{i=1}^{k} \xi_{i}\right\|$. Suppose $\left\|\sum_{i=1}^{k} x_{i}\right\|>k(1-\delta)$. Then $\left\|\sum_{i=1}^{k} \xi_{i}\right\|>$ $k\left(1-\delta_{3}\right)$. Then for each $j, 2 \leqq j \leqq k$,

$$
\begin{aligned}
\left\|\xi_{1}+\xi_{j}\right\| & \geqq\left\|\sum_{i=1}^{k} \xi_{i}\right\|-\left\|\sum_{i=2, i \neq j}^{k} \xi_{i}\right\| \\
> & k\left(1-\delta_{3}\right)-(k-2) \\
& =2\left(1-\frac{1}{2} k \delta_{3}\right)=2\left(1-\delta^{\prime}\left(\delta_{2}\right)\right) .
\end{aligned}
$$

Hence by the properties of $\delta^{\prime}(\cdot),\left\|\xi_{1}-\xi_{j}\right\|<\delta_{2}$, for each $j, 2 \leqq j \leqq k$. Since $\delta_{2}>0$, it is true that $\left\|\xi_{1}-\xi_{j}\right\|<\delta_{2}$ for $1 \leqq j \leqq k$.

We now define $z_{i} \in \mathfrak{Y}, 1 \leqq i \leqq k$, so that $z_{i}$ is a good approximation of $x_{i}$ and $\left\{z_{1}, \ldots, z_{k}\right\}$ satisfies the hypotheses of Part I of this proof. We let

$$
z_{i}(t)=\left\{\xi_{1}(t) / \xi_{i}(t)\right\} x_{i}(t)
$$


if $\xi_{i}(t) \neq 0$ and $z_{i}(t)=x_{1}(t)$ if $\xi_{i}(t)=0$. Then for each $t,\left\|z_{i}(t)\right\|=\xi_{1}(t)$, so $\left\|z_{i}\right\|=$ $\left\|\xi_{1}\right\| \leqq 1$ and $z_{1}, \ldots, z_{k}$ falls under Part I. Furthermore, if $\xi_{i}(t) \neq 0$,

$$
\begin{aligned}
\left\|z_{i}(t)-x_{i}(t)\right\| & =\left\|\left\{\xi_{1}(t) / \xi_{i}(t)\right\} x_{i}(t)-x_{i}(t)\right\| \\
& =\left\|\left(\left\{\xi_{1}(t) / \xi_{i}(t)\right\}-1\right) x_{i}(t)\right\|=\left|\left\{\xi_{1}(t) / \xi_{i}(t)\right\}-1\right| \cdot\left\|x_{i}(t)\right\| \\
& =\left|\xi_{1}(t)-\xi_{i}(t)\right|,
\end{aligned}
$$

and if $\xi_{i}(t)=0$,

$$
\begin{aligned}
\left\|z_{i}(t)-x_{i}(t)\right\| & =\left\|x_{1}(t)-0\right\| \\
& =\left|\xi_{1}(t)\right|=\left|\xi_{1}(t)-\xi_{i}(t)\right|
\end{aligned}
$$

so by componentwise comparison, $\left\|z_{i}-x_{i}\right\|=\left\|\xi_{1}-\xi_{i}\right\|<\delta_{2}$.

By Part I, choose $\lambda_{i}= \pm 1,1 \leqq i \leqq k$, so that $k^{-1}\left\|\sum_{i=1}^{k} \lambda_{i} z_{i}\right\| \leqq 1-\delta_{1}$. Then

$$
\begin{aligned}
k^{-1}\left\|\sum_{i=1}^{k} \lambda_{i} x_{i}\right\| & \leqq k^{-1}\left\|\sum_{i=1}^{k} \lambda_{i} z_{i}\right\|+k^{-1}\left\|\sum_{i=1}^{k} \lambda_{i}\left(z_{i}-x_{i}\right)\right\| \\
& \leqq 1-\delta_{1}+k^{-1} \sum_{i=1}^{k}\left|\lambda_{i}\right| \cdot\left\|z_{i}-x_{i}\right\| \\
& \leqq 1-\delta_{1}+k^{-1} \sum_{i=1}^{k} \delta_{2} \\
& =1-\left(\delta_{1}-\delta_{2}\right) \leqq 1-\delta .
\end{aligned}
$$

Thus, for $x_{1}, \ldots, x_{k} \in \mathfrak{Y},\left\|x_{i}\right\| \leqq 1$, there exist $\lambda_{i}= \pm 1$ such that

$$
k^{-1}\left\|\sum_{i=1}^{k} \lambda_{i} x_{i}\right\| \leqq 1-\delta .
$$

Thus $\mathfrak{Y}$ is $k, \delta$-convex. Q.E.D.

18. COROLlaRY. Let $\mathfrak{X}$ be a uniformly convex proper space of functions on an index set $\mathscr{T}$. For each $t \in \mathscr{T}$, let $\mathfrak{X}_{t}$ be a $k_{t}$, $\varepsilon_{t}$-convex $N L S$. Suppose $\sup _{\mathscr{T}} k_{t}=k<\infty$ and $\inf _{\mathscr{g}} \varepsilon_{t}=\varepsilon>0$. Then $P_{\mathfrak{X}} \mathfrak{X}_{t}$ is $k, \delta$-convex for suitable $\delta>0$.

Proof. We will reduce this to the previous theorem by showing that all $\mathfrak{X}_{t}$ are $k, \varepsilon^{\prime}$-convex for some $\varepsilon^{\prime}>0$.

Let us fix our attention on a fixed $t$ and let $k_{t}=n, \varepsilon_{t}=\theta$. $n \leqq k$, so write $k=a n+r$ $a>0,0 \leqq r<n$. Let $x_{1}, \ldots, x_{k} \in X_{t},\left\|x_{i}\right\| \leqq 1$. Dividing the $x_{i}$ into $a$ groups of $n$ elements and $r$ single elements, we fit each group with \pm 1 's so that the sum has 
norm $\leqq n(1-\theta)$. Thus using the triangle inequality, there are $\lambda_{i}= \pm 1,1 \leqq i \leqq k$, such that

$$
\left\|\sum_{i=1}^{k} \lambda_{i} x_{i}\right\| \leqq \operatorname{an}(1-\theta)+r=\operatorname{an}(1-\theta)+r(1-\theta)+r \theta=k(1-\theta)+r \theta .
$$

Clearly $r<k / 2$, so

$$
k^{-1}\left\|\sum_{i=1}^{k} \lambda_{i} x_{i}\right\| \leqq 1-\theta+(r / k) \theta<1-\frac{1}{2} \theta=1-\frac{1}{2} \varepsilon_{t} .
$$

Thus, $\mathfrak{X}_{t}$ is $k$, $\frac{1}{2} \varepsilon_{t}$-convex for $t \in \mathscr{T}$. Since $\varepsilon^{\prime}=\frac{1}{2} \varepsilon \leqq \frac{1}{2} \varepsilon_{t}, \mathfrak{X}_{t}$ is $k, \varepsilon^{\prime}$-convex for $t \in \mathscr{T}$. Thus Theorem 17 applies. Q.E.D.

19. TheOREM. A NLS $\mathfrak{X}$ is $k$, $\varepsilon$-convex (B-convex) if and only if each $k$ dimensional (separable) subspace is $k$, $\varepsilon$-convex (B-convex).

Proof. If $\mathfrak{X}$ is $k, \varepsilon$-convex ( $B$-convex) then every subspace of $\mathfrak{X}$ is $k, \varepsilon$-convex (B-convex). If $\mathfrak{X}$ is not $k, \varepsilon$-convex, there exist $x_{1}, \ldots, x_{k} \in S$ such that

$$
\left\| \pm x_{1} \pm x_{2} \pm \cdots \pm x_{k}\right\|>k(1-\varepsilon)
$$

for all choices of + and - signs. If we denote the span of $\left\{x_{1}, \ldots, x_{k}\right\}$ by $\mathfrak{X}(k, \varepsilon)$, then $\mathfrak{X}(k, \varepsilon)$ is a subspace of $\mathfrak{X}$ of at most dimension $k$ such that $\mathfrak{X}(k, \varepsilon)$ is not $k$, $\varepsilon$-convex. If $\mathfrak{X}$ is not $B$-convex, then for all $k \geqq 2, n \geqq 1, \mathfrak{X}$ is not $k, n^{-1}$-convex. Choosing $\mathfrak{X}\left(k, n^{-1}\right)$ as above and letting $\mathfrak{X}_{1}=\operatorname{span}\left\{\mathfrak{X}\left(k, n^{-1}\right): k \geqq 2, n \geqq 1\right\}$, then $\mathfrak{X}_{1}$ is separable and $\mathfrak{X}_{1}$ is not $B$-convex. Q.E.D.

III. Internal structure of $B$-convex spaces is explored in this section. We strengthen one direction of Beck's theorem, get a cancellation result much stronger in one sense than that of Lemma I.4, and discover a condition under which $B$-convex spaces are reflexive. We close with a curious example.

For definitions of various concepts involved in Beck's theorem, the reader is referred to the introduction of this paper, or to Beck [1] or [2].

1. Lemma. Let $(\Omega, \Sigma, m)$ be a measure space with $m(\Omega)<\infty$. Let $1 \leqq p<q \leqq \infty$. Let $\left\{f_{n}\right\}$ be a sequence of real valued functions on $\Omega$ such that

(i) $f_{n} \rightarrow 0$ almost everywhere or in measure,

(ii) $\left\|f_{n}\right\|_{q}$ is bounded uniformly in $n$.

Then $\lim _{n}\left\|f_{n}\right\|_{p}=0$.

REMARKs. As usual, $\left\|f_{n}\right\|_{p}=\left(\int_{\Omega}\left|f_{n}(\omega)\right|^{p} m d \omega\right)^{1 / p},\left\|f_{n}\right\|_{\infty}=\operatorname{ess}_{\Omega} \sup \left\|f_{n}(\omega)\right\|$. This result is well known. See, e.g., Loève [15, p. 164, Corollary 2]. 
2. TheOREM. Let $1 \leqq p<q \leqq \infty$ with $2 \leqq q$. Let $k \geqq 2, \varepsilon>0$. Then there exists $a$ sequence of real numbers $b_{n}=b_{n}(p, q, k, \varepsilon)$ such that $\lim _{n} b_{n}=0$ and if $\mathfrak{X}$ is a $k, \varepsilon-$ convex NLS, $(\Omega, \Sigma, \mathscr{P})$ is a probability space, and $X_{1}, \ldots, X_{n}$ are independent $\mathfrak{X}$-valued random variables on $\Omega$, with $E\left(X_{i}\right)=0$ and $\left\|X_{i}\right\|_{q} \leqq M, 1 \leqq i \leqq n$, then $\left\|n^{-1} \sum_{i=1}^{n} X_{i}\right\|_{p} \leqq M b_{n}$.

REMARKs. Here we use the notation $\left\|X_{i}\right\|_{p}=\left(\int_{\Omega}\left\|X_{i}\right\|^{p} d \mathscr{P}\right)^{1 / p}$ for $1 \leqq p<\infty$, $\left\|X_{i}\right\|_{\infty}=\operatorname{ess}_{\Omega} \sup \left\|X_{i}(\omega)\right\|$. This author first proved the special case of this theorem for $p=1, q=2$. Anatole Beck noticed that it could be generalized to all $p \in[1,2)$, and suggested a generalization which this author refined to the present form.

Specializing this theorem to the case of real- and complex-valued random variables, we find that for $1 \leqq p<q \leqq \infty$ and $q \geqq 2$ there exists a sequence $b_{n}=b_{n}(p, q)$ such that $\lim _{n} b_{n}=0$ and if $\left\{f_{n}\right\}$ is an independent sequence of random variables with $\left\|f_{n}\right\|_{q} \leqq M$ for all $n$, then $\left\|(1 / n) \sum_{i=1}^{n} f_{i}\right\|_{p} \leqq M b_{n}$. This shows that the speed of approach to 0 given by the strong law of large numbers is uniform over a wide class of sequences.

Proof. For a fixed $p$ and $q$ with $1 \leqq p<q \leqq \infty$ and $q \geqq 2$, let $\mathfrak{X}$ be an arbitrary $B$-convex NLS, $(\Omega, \Sigma, \mathscr{P})$ a probability space and $\left\{X_{n}\right\}$ an independent sequence of $\mathfrak{X}$-valued random variables with $E\left(X_{n}\right)=0$ and $\left\|X_{n}\right\|_{q} \leqq M$ for all $n$. Let

$$
E_{n}=\left\{\omega \in \Omega:\left\|X_{n}(\omega)\right\| \leqq 1\right\}
$$

$F_{n}=\Omega-E_{n}$. If $q<\infty$,

$$
\begin{aligned}
\operatorname{var}\left(X_{n}\right) & =\left\|X_{n}\right\|_{2}^{2}=\int_{\Omega}\left\|X_{n}\right\|^{2} d \mathscr{P}=\int_{E_{n}}\left\|X_{n}\right\|^{2} d \mathscr{P}+\int_{F_{n}}\left\|X_{n}\right\|^{2} d \mathscr{P} \\
& \leqq 1+\int_{F_{n}}\left\|X_{n}\right\|^{q} d \mathscr{P} \leqq 1+\int_{\Omega}\left\|X_{n}\right\|^{q} d \mathscr{P} \leqq 1+M^{q},
\end{aligned}
$$

and if $q=\infty$,

$$
\begin{aligned}
\operatorname{var}\left(X_{n}\right) & =\int_{\Omega}\left\|X_{n}\right\|^{2} d \mathscr{P} \leqq \operatorname{ess}_{\Omega} \sup \left\|X_{n}\right\|^{2}=\left(\operatorname{ess}_{\Omega} \sup \left\|X_{n}\right\|\right)^{2} \\
& =\left\|X_{n}\right\|_{\infty}^{2} \leqq M^{2}
\end{aligned}
$$

so var $\left(X_{n}\right)$ is uniformly bounded, hence by Beck's theorem,

$$
\lim _{n}\left\|n^{-1} \sum_{i=1}^{n} X_{i}(\omega)\right\|=0
$$

for almost all $\omega .\left\|n^{-1} \sum_{i=1}^{n} X_{i}\right\|_{q} \leqq n^{-1} \sum_{i=1}^{n}\left\|X_{i}\right\|_{q} \leqq M$, so by Lemma 1,

$$
\lim _{n}\left\|n^{-1} \sum_{i=1}^{n} X_{i}\right\|_{p}=0
$$


Now, fix $k \geqq 2$ and $\varepsilon>0$. Define $b_{n}=\sup \left\|n^{-1} \sum_{i=1}^{n} X_{i}\right\|_{p}$ where the sup extends over all $k, \varepsilon$-convex Banach spaces $\mathfrak{X}$, all probability spaces $(\Omega, \Sigma, \mathscr{P})$, and all independent sequences $X_{1}, \ldots, X_{n}$ of $\mathfrak{X}$-valued random variables on $\Omega$ with $E\left(X_{i}\right)=0$ and $\left\|X_{i}\right\|_{q} \leqq 1,1 \leqq i \leqq n$. Then $b_{n}$ depends only on $p, q, k$, and $\varepsilon$ and $0 \leqq b_{n} \leqq \infty$. Let $\lim _{n} \sup b_{n}=\alpha$. Then $0 \leqq \alpha \leqq \infty$. We wish to show $\alpha=0$.

Suppose $\alpha>0$. Choose $\beta>0$ such that $2 \beta<\alpha$. We will now construct an independent sequence of $\mathfrak{X}$-valued random variables $\left\{X_{n}\right\}$ on $\Omega$, where $E\left(X_{i}\right)=0$ and $\left\|X_{i}\right\|_{q} \leqq 1$, all $i,(\Omega, \Sigma, \mathscr{P})$ is a probability space, and $\mathfrak{X}$ is $B$-convex, having the property that $\lim _{n} \sup \left\|n^{-1} \sum_{i=1}^{n} X_{i}\right\|_{p} \geqq \beta>0$, contradicting the result we got in the first part of this proof.

Using the fact that $\lim _{n}$ sup $b_{n}>2 \beta$, for each $n$ pick an integer $m(n) \geqq n$, a $k, \varepsilon$ convex NLS $\mathfrak{X}_{n}$, a probability space $\left(\Omega_{n}, \Sigma_{n}, \mathscr{P}_{n}\right)$, and an independent sequence of $\mathfrak{X}_{n}$-valued random variables on $\Omega_{n}, Y_{1}^{(n)}, \ldots, Y_{m(n)}^{(n)}$ such that $E\left(Y_{i}^{(n)}\right)=0$ and $\left\|Y_{i}^{(n)}\right\|_{q} \leqq 1$ and $\left\|m(n)^{-1} \sum_{i=1}^{m(n)} Y_{i}^{(n)}\right\|_{p} \geqq 2 \beta$.

Let $\mathfrak{X}=P_{\ell_{2}} \mathfrak{X}_{n}$ and $(\Omega, \Sigma, \mathscr{P})=\prod_{n=1}^{\infty}\left(\Omega_{n}, \Sigma_{n}, \mathscr{P}_{n}\right)$. Then $\mathfrak{X}$ is $B$-convex by Theorem II.17 and Example I.3(ii), and $(\Omega, \Sigma, \mathscr{P})$ is a probability space. For all $n$ and $1 \leqq i \leqq m(n)$ define $X_{i}^{(n)}$ on $\Omega$ into $\mathfrak{X}$ by $X_{i}^{(n)}\left(\omega_{1}, \omega_{2}, \ldots\right)=\left(x_{1}, x_{2}, \ldots\right)$ where $x_{n}=Y_{i}^{(n)}\left(\omega_{n}\right)$ and $x_{j}=0$ for $j \neq n$. Then for all $n$ and $1 \leqq i \leqq m(n), E\left(X_{i}^{(n)}\right)=0$ and $\left\|X_{i}^{(n)}\right\|_{q} \leqq 1$, and $\left\|m(n)^{-1} \sum_{i=1}^{m(n)} X_{i}^{(n)}\right\|_{p}=\left\|m(n)^{-1} \sum_{i=1}^{m(n)} Y_{i}^{(n)}\right\|_{p} \geqq 2 \beta$, and

$$
\left\{X_{i}^{(n)}: 1 \leqq i \leqq m(n), n \geqq 1\right\}
$$

is an independent set of $\mathfrak{X}$ valued random variables on $\Omega$.

Let $n_{1}=1, r_{1}=m\left(n_{1}\right)$ and $X_{i}=X_{i}^{\left(n_{1}\right)}$ for $1 \leqq i \leqq m\left(n_{1}\right)$. Then $\left\|r_{1}^{-1} \sum_{i=1}^{r_{i}} X_{i}\right\|_{p} \geqq$ $2 \beta>\beta$. Suppose we have chosen $r_{1}<r_{2}<\cdots<r_{s}$ and a sequence $X_{i}, 1 \leqq i \leqq r_{s}$, such that $\left\|r_{j}^{-1} \sum_{i=1}^{r_{j}} X_{i}\right\|_{p} \geqq \beta$ for $1 \leqq j \leqq s$. Let $\gamma_{s}=\left\|r_{s}^{-1} \sum_{i=1}^{r_{s}} X_{i}\right\|_{p}$. Choose $n_{s+1}$ so large that if we let $m_{s+1}=m\left(n_{s+1}\right)$, then

$$
\left(2 m_{s+1}-r_{s}\left(\gamma_{s} / \beta\right)\right) /\left(m_{s+1}+r_{s}\right) \geqq 1 .
$$

For $1 \leqq i \leqq m\left(n_{s+1}\right)$, define $X_{r_{s}+i}=X_{i}^{\left(n_{s+1}\right)}$. Let $r_{s+1}=m\left(n_{s+1}\right)+r_{s}$. Then $\left(^{*}\right)$ becomes

$$
\left(2 m_{s+1}-r_{s}\left(\gamma_{s} / \beta\right)\right) / r_{s+1} \geqq 1
$$

$$
\begin{aligned}
\left\|r_{s+1}^{-1} \sum_{i=1}^{r_{s+1}} X_{i}\right\|_{p} & \geqq r_{s+1}^{-1}\left\|_{i=r_{s}+1}^{r_{s+1}} X_{i}\right\|_{p}-r_{s+1}^{-1}\left\|\sum_{i=1}^{r_{s}} X_{i}\right\|_{p} \\
& =\left(m_{s+1} / r_{s+1}\right) m_{s+1}^{-1}\left\|\sum_{i=1}^{m_{s+1}} X_{i}^{\left(n_{s+1}\right)}\right\|_{p}-\left(r_{s} / r_{s+1}\right) r_{s}^{-1}\left\|\sum_{i=1}^{r_{s}} X_{i}\right\|_{p} \\
& \geqq\left(m_{s+1} / r_{s+1}\right)(2 \beta)-\left(r_{s} / r_{s+1}\right) \gamma_{s} \\
& =\beta\left(2 m_{s+1}-r_{s}\left(\gamma_{s} / \beta\right)\right) / r_{s+1} \geqq \beta
\end{aligned}
$$

by $(* *)$. 
Hence by induction, we have chosen from the independent set

$$
\left\{X_{i}^{(n)}: 1 \leqq i \leqq m(n), n \geqq 1\right\}
$$

of $\mathfrak{X}$-valued random variables with 0 expectation and $q$-norms uniformly bounded by 1 , a sequence $X_{n}$ such that $\lim _{n}$ sup $\left\|n^{-1} \sum_{i=1}^{n} X_{i}\right\|_{p} \geqq \beta$, a contradiction.

Hence $\lim _{n} b_{n}=0$. To complete the proof, let $\mathfrak{X}$ be a $k, \varepsilon$-convex NLS, $(\Omega, \Sigma, \mathscr{P})$ a probability space, and $\left\{X_{n}\right\}$ a sequence of $\mathfrak{X}$-valued random variables on $\Omega$ with $E\left(X_{n}\right\}=0$ and $\left\|X_{n}\right\|_{q} \leqq M$ for all $n$. Hence $\left\|M^{-1} X_{n}\right\|_{q} \leqq 1$. Thus, the independent sequence $\left\{M^{-1} X_{n}\right\}$ satisfies $E\left(M^{-1} X_{n}\right)=0$ and $\left\|M^{-1} X_{n}\right\|_{q} \leqq 1$, so

$$
\left\|n^{-1} \sum_{i=1}^{n} M^{-1} X_{n}\right\|_{p} \leqq b_{n}
$$

hence $\left\|n^{-1} \sum_{i=1}^{n} X_{n}\right\|_{p} \leqq M b_{n}$ for all $n$. Q.E.D.

3. THEOREM. Let $p \geqq 1, k \geqq 2$, and $\varepsilon>0$ and let $b_{n}=b_{n}(p, \infty, k, \varepsilon)$ be the sequence of Theorem 2. Let $\left(\Omega_{n}, \Sigma_{n}, \mu_{n}\right)$ be a probability space, $f_{n}$ a $D$-valued measurable function on $\Omega_{n}$ such that $\int_{\Omega_{n}} f_{n} d \mu_{n}=0$. Let $(\Omega, \Sigma, \mu)=\prod_{n=1}^{\infty}\left(\Omega_{n}, \Sigma_{n}, \mu_{n}\right)$ and define $g_{n}$ on $\Omega$ by $g_{n}\left(\omega_{1}, \omega_{2}, \ldots\right)=f_{n}\left(\omega_{n}\right)$. Then $\int_{\Omega} g_{n} d \mu=0$. Let $\mathfrak{X}$ be a $k, \varepsilon$-convex $N L S$, and let $\left\{x_{n}\right\} \subset S$. Then

(1) $\lim _{n} n^{-1}\left\|\sum_{i=1}^{n} g_{n}(\omega) x_{n}\right\|=0$ for almost all $\omega \in \Omega$, and

(2) $\left(\int_{\Omega}\left\|n^{-1} \sum_{i=1}^{n} g_{n} x_{n}\right\|^{p} d \mu\right)^{1 / p} \leqq b_{n}$.

Proof. $\left\{g_{n}\right\}$ is an independent sequence of $D$-valued random variables on $\Omega$. If we define $X_{n}(\omega)=g_{n}(\omega) x_{n}$, then $\left\{X_{n}\right\}$ is an independent sequence of $\mathfrak{X}$-valued random variables. We have $E\left(X_{n}\right)=\int_{\Omega} g_{n}(\omega) x_{n} d \mu=x_{n} \int_{\Omega} g_{n}(\omega) d \mu=0$ and $\left\|X_{n}\right\|_{\infty}$ $\leqq 1$ so by Beck's theorem, $\lim _{n} n^{-1}\left\|\sum_{i=1}^{n} X_{i}(\omega)\right\|=0$ for almost all $\omega$ and by Theorem 2, $\left\|n^{-1} \sum_{i=1}^{n} X_{i}\right\|_{p} \leqq b_{n}$. Hence $\lim _{n} n^{-1}\left\|\sum_{i=1}^{n} g_{i}(\omega) x_{i}\right\|=0$ for almost all $\omega$ and $\left(\int_{\Omega}\left\|n^{-1} \sum_{i=1}^{n} g_{i}(\omega) x_{i}\right\|^{p} d \mu\right)^{1 / p} \leqq b_{n}$. Q.E.D.

4. Comments. Lemma I.4 showed that, for a $k$, $\varepsilon$-convex NLS $\mathfrak{X}$ and under the condition that $A$ was closed under multiplication, for every $\varepsilon>0$ there exists $N$ such that if $n \geqq N$, and $x_{1}, \ldots, x_{n} \in S$, then for some choice of $\lambda_{1}, \ldots, \lambda_{n} \in A$, $n^{-1}\left\|\sum_{i=1}^{n} \lambda_{i} x_{i}\right\| \leqq \varepsilon$. Theorem 3 shows us that for a large class $\mathfrak{M}=\{\mu\}$ of measures on $D^{\infty}=\left\{\lambda=\left(\lambda_{1}, \lambda_{2}, \ldots\right): \lambda_{i} \in D\right\}$, if $x_{1}, x_{2}, \ldots \in S$ and $\lambda \in D^{\infty}$,

$$
f_{n}(\lambda)=n^{-1}\left\|\sum_{i=1}^{n} \lambda_{i} x_{i}\right\|,
$$

then $f_{n} \rightarrow 0$ almost everywhere $[\mu]$ and $\left\|f_{n}\right\|_{p} \leqq b_{n}(p, \infty, k, \varepsilon)$, so $f_{n} \rightarrow 0$ in the $\mu$-mean of order $p \geqq 1$ uniformly for all sequences $x_{1}, x_{2}, \ldots \in S$, for all $k, \varepsilon$-convex $\mathfrak{X}$, and for all $\mu \in \mathfrak{M}$. The only recommendation for Lemma I.4 at this point is its 
comparatively simple and intuitive proof from the definitions involved while Theorem 3 rests on Beck's theorem whose proof is tremendously involved, and the estimate $a_{n}(\mathfrak{X}, A)=O\left(n^{-\gamma}\right)$ where the $O$-constant and $\gamma$ depend only on $k$ and $\varepsilon$ while nothing is known of the speed of convergence of $b_{n}(p, q, k, \varepsilon)$ to 0 .

A sequence of measure spaces $\left(\Omega_{n}, \Sigma_{n}, \mu_{n}\right)$ gives rise to a direct product measure space $(\Omega, \Sigma, \mu)=\left(\Pi_{n} \Omega_{n}, \Pi_{n} \Sigma_{n}, \Pi_{n} \mu_{n}\right)$. Conversely, if $(\Omega, \Sigma, \mu)$ is a measure space and $\Omega=\Pi_{n} \Omega_{n}$, then $(\Omega, \Sigma, \mu)$ induces a $\sigma$-field $\Sigma_{n}$ and a measure $\mu_{n}$ on $\Omega_{n}$ by $\Sigma_{n}=\left\{\Gamma_{n} \subset \Omega_{n}: \Gamma=\Omega_{1} \times \cdots \times \Omega_{n-1} \times \Gamma_{n} \times \Omega_{n+1} \times \cdots \in \Sigma\right\}$ and $\mu_{n}\left(\Gamma_{n}\right)=\mu(\Gamma)$.

Let $\mu$ be a measure on $D^{\infty}$ and let $\mu_{n}$ be the measure induced by $\mu$ on the $n$th component of $D^{\infty}$. Call $\mu_{n}$ admissible if $\mu_{n}(D)=1$ and $\int_{D} \lambda d \mu_{n}=0$. (If an admissible $\mu_{n}$ is considered to be a mass distribution on $D$, then $D$ has mass 1 and center of gravity 0.) Call $\mu$ admissible if all $i_{n}$ are admissible. Let $\mathfrak{M}=\{\mu: \mu$ is admissible $\}$.

Let $\mu \in \mathfrak{M}$. Then $\left(D, \Sigma_{n}, \mu_{n}\right)$ is a probability space for each $n$. Let $f_{n}$ be the identity function on $D$. Then $f_{n}$ satisfies the hypotheses of Theorem 3 with $\Omega_{n}=D$. Let $\mathfrak{X}$ be a $k, \varepsilon$-convex NLS and let $\left\{x_{n}\right\} \subset S$. Then, by Theorem 3,

$$
\lim _{n} n^{-1}\left\|\sum_{i=1}^{n} \lambda_{i} x_{i}\right\|=0
$$

for $\mu$-almost all $\lambda \in D^{\infty}$ and $\left\|n^{-1} \sum_{i=1}^{n} \lambda_{i} x_{i}\right\|_{p} \leqq b_{n}(p, \infty, k, \varepsilon)$. Thus, the assertions of the early part of this comment are established.

In the case $D=B$, the only admissible measure on $D$ is $\mu_{n}(\{1\})=\mu_{n}(\{-1\})=\frac{1}{2}$. In this case, $\int_{D^{\infty}} n^{-1}\left\|\sum_{i=1}^{n} \lambda_{i} x_{i}\right\| d \mu=2^{-n} \sum_{\lambda_{1}= \pm 1,1 \leqq i \leqq n} n^{-1}\left\|\sum_{i=1}^{n} \lambda_{i} x_{i}\right\|$. Thus, as $n$ gets large not only is one of the numbers $n^{-1}\left\| \pm x_{1} \pm x_{2} \pm \cdots \pm x_{n}\right\|$ small as asserted by Lemma I.4, but the average of all of the nonnegative numbers

$$
n^{-1}\left\| \pm x_{1} \pm x_{2} \pm \cdots \pm x_{n}\right\|
$$

is small, hence, most of them are small.

5. Corollary. Let $\mathfrak{X}$ be a B-convex $N L S$. Then $\lim _{n} a_{n}(\mathfrak{X}, A)=0$ if and only if 0 is in the closed convex hull of $A$ in the complex plane.

Proof. For the convex hull of $A$, write $\operatorname{co}(A)$, for the closed convex hull of $A$, write $\operatorname{cl}(\operatorname{co}(A))$. In the remarks following the proof of Lemma I.4, we showed that if $0 \notin \mathrm{cl}(\operatorname{co}(A))$, say $d(0, \operatorname{cl}(\operatorname{co}(A)))=\beta>0$, then $\lim _{n} \inf a_{n}(\mathfrak{X}, A) \geqq \beta$. We prove the converse here.

By Remark I.2(c2) if $\bar{A}$ denotes the closure of $A, a_{n}(\mathfrak{X}, A)=a_{n}(\mathfrak{X}, \bar{A})$, so it suffices to consider closed sets $A$. Since $A$ is a closed subset of $C, A$ is compact. Since $0 \in \operatorname{cl}(\operatorname{co}(A))$, there is a sequence $\left\{x_{n}\right\} \subset \operatorname{co}(A)$ such that $\lim _{n} x_{n}=0$. By a theorem of Carathéodory (cf. e.g., Eggleston [8, p. 35]), every point of the convex hull of a set in the plane is a linear combination of 3 points of that set, so there are $\lambda_{i}(n) \in A$ and $\alpha_{i}(n) \in[0,1], i=1,2,3$, such that $\Sigma_{i} \alpha_{i}(n)=1$ and $\Sigma_{i} \alpha_{i}(n) \lambda_{i}(n)=x_{n}$. Choose a 
subsequence $n_{j}$ such that $\alpha_{i}\left(n_{j}\right) \rightarrow \alpha_{i}$ and $\lambda_{i}\left(n_{j}\right) \rightarrow \lambda_{i}$, for $i=1,2,3$. Then $\alpha_{i} \in[0,1]$ and $\lambda_{i} \in A$ for $i=1,2,3$, and $\Sigma_{i} \alpha_{i}=1$ and $\Sigma_{i} \alpha_{i} \lambda_{i}=0$. Let $\Omega_{n}=\left\{\lambda_{i}: 1 \leqq i \leqq 3\right\}$, $\Sigma_{n}=2^{\Omega_{n}}$, and define $\mu_{n}$ by $\mu_{n}\left(\left\{\lambda_{i}\right\}\right)=\alpha_{i}$. If $f_{n}\left(\lambda_{i}\right)=\lambda_{i}, i=1,2,3$, then the hypotheses of Theorem 3 are satisfied, so for $x_{1}, \ldots, x_{n} \in S, \int_{\Omega} n^{-1}\left\|\sum_{i=1}^{n} g_{n}(\omega) x_{n}\right\| d \mu \leqq b_{n}$. Hence for some $\omega \in \Omega, n^{-1}\left\|\sum_{i=1}^{n} g_{n}(\omega) x_{n}\right\| \leqq b_{n}$. Hence for some sequence $\zeta_{1}, \ldots, \zeta_{n}$ from $\left\{\lambda_{1}, \lambda_{2}, \lambda_{3}\right\}, n^{-1}\left\|\sum_{i=1}^{n} \zeta_{n} x_{n}\right\| \leqq b_{n}$, so that $a_{n}(\mathfrak{X}, A) \leqq b_{n}$. Hence $\lim _{n} a_{n}(\mathfrak{X}, A)=0$. Q.E.D.

Remark. Let $A \subset D$. If we define an admissible measure on $A$ to be an admissible measure $\mu$ on $D$ such that $\mu(D-A)=0$, then the preceding argument shows that for a closed set $A$, there exists an admissible measure on $A$ if and only if $0 \in \operatorname{cl}(\operatorname{co}(A))$.

6. THEOREM. Let $\mathfrak{X}$ be a B-convex Banach space with an unconditional basis. Then $\mathfrak{X}$ is reflexive.

Remarks. This theorem is due independently to R. C. James [13] and this author.

A basis for a Banach space $\mathfrak{X}$ is a sequence $\left\{x_{n}\right\} \subset \mathfrak{X}$ such that if $x \in \mathfrak{X}$, there exists a unique sequence $\left\{\alpha_{n}\right\}$ of scalars such that $\lim _{n}\left\|x-\sum_{i=1}^{n} \alpha_{i} x_{i}\right\|=0$. We write $x=\sum_{i=1}^{\infty} \alpha_{i} x_{i}$.

Let $\Sigma$ be the set of all finite subsets of the positive integers directed by set inclusion. A basis $\left\{x_{n}\right\}$ of $\mathfrak{X}$ is unconditional if for $x \in \mathfrak{X}, x=\sum_{i=1}^{\infty} \alpha_{i} x_{i}$, we have $\lim _{\sigma \in \Sigma}\left\|x-\sum_{i \in \sigma} \alpha_{i} x_{i}\right\|=0$.

By a theorem of James [11], a space $\mathfrak{X}$ with an unconditional basis is reflexive if and only if no subspace of $\mathfrak{X}$ is isomorphic to $\ell_{1}$ or $c_{0}$.

Proof. Since $\mathfrak{X}$ is $B$-convex, every subspace of $\mathfrak{X}$ is $B$-convex by Remark I.2(c1). Neither $\ell_{1}$ nor $c_{0}$ is $B$-convex (Examples I.3(iii) and (iv) respectively). Hence, by Corollary II.6, no isomorph of $\ell_{1}$ or $c_{0}$ is $B$-convex. Hence no subspace of $\mathfrak{X}$ can be isomorphic to $\ell_{1}$ or $c_{0}$. Therefore, by James' theorem, $\mathfrak{X}$ is reflexive. Q.E.D.

7. EXAMPLE. In the introduction we mentioned that $B$-convexity and in particular 2, $\varepsilon$-convexity, locally uniform convexity and reflexivity were all generalizations of uniform convexity. We give here an example of a $2, \delta$-convex, locally uniformly convex, reflexive space which is not uniformly convex. It would be much more impressive if it were not isomorphic to a uniformly convex space; however, this is not the case.

We will construct a sequence $\left\{\mathfrak{X}_{n}\right\}$ of 2-dimensional, 2, $\varepsilon$-convex, locally uniformly convex, reflexive spaces and let $\mathfrak{X}=P_{\ell_{2}} \mathfrak{X}_{n}$. Then $\mathfrak{X}$ will be $2, \delta$-convex (by Theorem II.17), locally uniformly convex (Lovaglia [16]), and reflexive (Day [6, p. 31]). However $\left\{\mathfrak{X}_{n}\right\}$ will be constructed so that $\mathfrak{X}$ is not uniformly convex.

For ease of description, we need the following definitions. If $A$ is a subset of a linear space and $\alpha$ is a scalar, $\alpha A=\{\alpha x: x \in A\} . A$ is symmetric about 0 if $A=(-1) A$. $A$ is linearly bounded (closed) if the intersection of $A$ with each one-dimensional subspace is bounded (closed) in the usual topology of the line. For each vector $x$, 
$[-x, x]$ denotes the line segment joining $x$ and $-x .0$ is an internal point of $A$ if for every vector $x$ there exists $\varepsilon>0$ such that $\varepsilon[-x, x] \subset A$. $A$ is called a sphere set if it is convex, symmetric about 0 , and linearly bounded and closed, and 0 is an internal point of $A$. The Minkowski functional of a sphere set $A$ is a norm whose closed unit sphere is $A$; all closed unit spheres in NLS's are sphere sets.

Let $A$ be a sphere set and $\|\cdot\|$ the norm whose unit sphere is $A$. Then $x \in A$ if and only if $\|x\| \leqq 1 . A$ is rotund if for every $x$ and $y$ in $A$ with $x \neq y,\|x+y\|<2$; equivalently, the boundry of $A$ contains no line segments.

Let $C$ be the Euclidian unit circle in Euclidian two-space $E^{2}$. Let $S$ be a nonrotund sphere set in $E^{2}$ such that $S \subset C$ and there exists $\varepsilon>0$ such that $(\sqrt{ } 1 / 2) C \subset(1-\varepsilon) S$ (for example $S$ could be a regular inscribed hexagon of $C$ ). Let $\left\{S_{n}\right\}$ be a sequence of rotund sphere sets in $E_{2}$ such that

$$
C \supset S_{1} \supset S_{2} \supset \ldots \supset S
$$

and $S=\bigcap_{n} S_{n}$ (for example, $S_{n}$ could be the result of replacing the sides of the hexagon by circular arcs of radius $n$ ). Let $\|\cdot\|$ be the Euclidian norm in $E^{2}$, $\|\cdot\|_{n}$ the norm generated by $S_{n}$ and $\|\cdot\|_{\infty}$ the norm generated by $S$. We should not confuse the norms defined here with the Lebesgue norms usually denoted in this fashion. Then for $x \in E^{2},\|x\| \leqq\|x\|_{1} \leqq\|x\|_{2} \leqq \cdots \leqq\|x\|_{\infty}$ and $\lim _{n}\|x\|_{n}=\|x\|_{\infty}$.

Let $\mathfrak{X}_{n}$ be the vector space $E^{2}$ with the norm generated by $S_{n}$. Since $\mathfrak{X}_{n}$ is finite dimensional and $S_{n}$ is rotund, a compactness argument shows $\mathfrak{X}_{n}$ is uniformly convex, hence locally uniformly convex. Since $\mathfrak{X}_{n}$ is finite dimensional, it is reflexive. Let $x$ and $y$ be in $S_{n}$. Since $\|\cdot\|$ is an inner product norm, the Jordan-von Neuman relation (parallelogram law) holds and

$$
\|x+y\|^{2}+\|x-y\|^{2}=2\|y\|^{2}+2\|x\|^{2} \leqq 4
$$

since $\|x\| \leqq\|x\|_{n} \leqq 1$ and $\|y\| \leqq\|y\|_{n} \leqq 1$. Hence for some choice of $\lambda= \pm 1$, $(1 / 2)\|x+\lambda y\| \leqq \sqrt{ } 1 / 2$. Since

$$
\begin{gathered}
(1 / 2)(x+\lambda y) \in(\sqrt{ } 1 / 2) C \subset(1-\varepsilon) S, \\
(1 / 2)\|x+\lambda y\|_{n} \leqq(1 / 2)\|x+\lambda y\|_{\infty} \leqq 1-\varepsilon,
\end{gathered}
$$

so $\mathfrak{X}_{n}$ is 2 , $\varepsilon$-convex.

Since $S$ is not rotund, we may choose $x$ and $y$ in $S$ such that $x \neq y$, hence $\|x-y\|=\eta>0,\|x\|_{\infty}=\|y\|_{\infty}=1$ and $\|x+y\|_{\infty}=2$.

Let $\mathfrak{X}=P_{\ell_{2}} \mathfrak{X}_{n}$. Then $\mathfrak{X}$ is $2, \delta$-convex, locally uniformly convex, and reflexive for reasons given above. Let $x_{n} \in \mathfrak{X}$ have all coordinates 0 except the $n$th coordinate, which is $x$. Define $y_{n} \in \mathfrak{X}$ from $y$ similarly.

$$
\left\|x_{n}-y_{n}\right\|_{\mathfrak{X}}=\|x-y\|_{n} \geqq\|x-y\|=\eta>0
$$


for all $n$, so if $\mathfrak{X}$ were uniformly convex there would exist $\theta>0$ such that $\left\|x_{n}+y_{n}\right\|_{\mathfrak{X}}<2(1-\theta)$ for all $n$. However

$$
\left\|x_{n}+y_{n}\right\|_{\mathfrak{X}}=\|x+y\|_{n} \rightarrow\|x+y\|_{\infty}=2
$$

as $n \rightarrow \infty$. Hence $\mathfrak{X}$ is not uniformly convex.

IV. Miscellany. This section is a compendium of conjectures, open questions, partial results, and acknowledgements.

1. Reflexivity. The principal outstanding conjecture about $B$-convex Banach spaces is that they are reflexive. There is a great deal of heuristic evidence to support this. James [13] has shown that all 2, $\varepsilon$-convex spaces are reflexive. He has also obtained partial results for the case $k=3$. Call $\mathfrak{X}$ strongly $3, \varepsilon$-convex if for each choice of $x, y, z \in S,\|x \pm y \pm z\| \leqq 3(1-\varepsilon)$ for at least 2 of the four choices of + and - signs. Then James has shown [13] that strongly $3, \varepsilon$-convex spaces are reflexive. James [13] and this author (Theorem III.6) have shown independently that $B$-convex spaces with unconditional bases are reflexive. Our Theorem II.1 shows a similarity in structure between a $k, \varepsilon$-convex $\mathfrak{X}$ and its $k, \varepsilon$-convex second conjugate. Since $k, \varepsilon$-convexity is invariant under isomorphism, we looked at an example of a nonreflexive space isometric to its second conjugate (Example I.7(ii)), and found that it was not $B$-convex. In fact, every nonreflexive space for which the question has been answered has turned out not to be $B$-convex. $B$ convexity parallels reflexivity in many theorems. Thus, given a space $\mathfrak{X}$ and a closed subspace $3, \mathfrak{X}$ is $B$-convex if and only if 8 and $\mathfrak{X} / 3$ both are (Theorem II.9), just as $\mathfrak{X}$ is reflexive if and only if 8 and $\mathfrak{X} / \mathbb{Z}$ both are (see, e.g., Dunford and Schwartz [7, II.4, 19-20]). $\mathfrak{X}$ is $B$-convex if and only if each separable subspace of $\mathfrak{X}$ is $B$-convex (Theorem II.19) just as $\mathfrak{X}$ is reflexive if and only if each separable subspace of $\mathfrak{X}$ is reflexive (see, e.g., Dunford and Schwartz [7, V. 4.7 and V. 6.1]).

In fact, it is still an open question whether or not all $B$-convex spaces are isomorphic to uniformly convex spaces. If they are, this would establish the Reflexivity Conjecture. This suggests the problem of determining for given $B$-convex $\mathfrak{X}$, what numbers $k$ and $\varepsilon$ there are for which some isomorph of $\mathfrak{X}$ is $k, \varepsilon$-convex. In particular, if every $B$-convex space is isomorphic to a $2, \varepsilon$-convex space, then James' theorem would tell us that every $B$-convex space is reflexive. Failing to establish the Reflexivity Conjecture would still leave open the following questions: Does a separable $B$-convex Banach space have a separable second (equivalently, first) conjugate? Is a $B$-convex Banach space weakly complete? (A sequence $\left\{x_{n}\right\} \subset \mathfrak{X}$ is weakly Cauchy if for each $x^{*} \in \mathfrak{X}^{*},\left\{x^{*}\left(x_{n}\right)\right\}$ is a Cauchy sequence of scalars. $\mathfrak{X}$ is weakly complete if each weakly Cauchy sequence $\left\{x_{n}\right\}$ has a weak limit $x$, i.e., $\lim _{n} x^{*}\left(x_{n}\right)=x^{*}(x)$ for each $x^{*} \in \mathfrak{X}^{*}$.)

2. $\mathfrak{B}(\mathfrak{X}, 3)$ is the Banach space of all continuous linear transformations of $\mathfrak{X}$ into 3 . Writing $\mathfrak{B}=\mathfrak{B}(\mathfrak{X}, \mathfrak{3})$, it is easily seen that $\mathfrak{B}$ contains isometric copies of 
$\mathfrak{X}^{*}$ and $\mathbb{Z}$, so in order that $\mathfrak{B}$ be $B$-convex, it is necessary that both $\mathfrak{X}$ and $\mathbb{Z}$ be $B$-convex. If $\mathfrak{X}$ is $n$-dimensional, $n<\infty$, and 3 is $B$-convex, $\mathfrak{B}$ is isomorphic to $\sum \oplus_{i=1}^{n} B_{i}$ (direct sum) where $3_{i}=3$, hence $\mathfrak{B}$ is $B$-convex. Similarly if $\mathfrak{X}$ is $B$ convex and 3 is finite dimensional, $\mathfrak{B}$ is isomorphic to the finite direct sum of copies of $\mathfrak{X}^{*}$, hence, is $B$-convex. However, in the case where both $\mathfrak{X}$ and 3 are infinite dimensional, the question is not so easily settled. Some of the "nicest" $B$-convex spaces are $\ell_{p}, 1<p<\infty$. However, we see $\mathfrak{B}\left(\ell_{p}, \ell_{p}\right)$ is not $B$-convex as follows: For $k \geqq 1$, let $x_{k}=\left(x_{k}(1), x_{k}(2), \ldots\right)$ be the sequence of +1 's and -1 's where $x_{k}(1)=+1$ and the +1 's and -1 's alternate in blocks of $2^{k-1}$ entries. Let $u_{1}, u_{2}, \ldots$ be the natural basis of $\ell_{p}$. Define $T_{k} \in \mathfrak{B}\left(\ell_{p}, \ell_{p}\right)$ by $T_{k}\left(u_{j}\right)=x_{k}(j) u_{j}$ and extend by linearity and continuity. It is easily seen that $T_{k}$ is an isometry, hence, in particular, $\left\|T_{k}\right\|=1$. If $\lambda=\left(\lambda_{1}, \ldots, \lambda_{k}\right)$ is a sequence of +1 's and -1 's, there exists $j$ such that $x_{i}(j)=\lambda_{i}, 1 \leqq i \leqq k$. Then $\sum_{i=1}^{k} \lambda_{i} T_{i}\left(u_{j}\right)=\sum_{i=1}^{k} \lambda_{i} x_{i}(j)=\sum_{i=1}^{k} \lambda_{i}^{2}=k$, so $\left\|\sum_{i=1}^{k} \lambda_{i} T_{i}\right\|=k$. Hence $\mathfrak{B}\left(\ell_{p}, \ell_{p}\right)$ is not $B$-convex.

It is conjectured that for $\mathfrak{X}$ and $\mathfrak{Y}$ both infinite dimensional, $\mathfrak{B}(\mathfrak{X}, \mathfrak{Y})$ is never $B$-convex. The technique given above can be generalized to show $\mathfrak{B}(\mathfrak{X}, \mathfrak{X})$ is not $B$-convex when $\mathfrak{X}$ has an unconditional basis or when $\mathfrak{X}$ is a proper space of functions. Beyond that point, nothing is known.

3. $P_{\mathfrak{X}} \mathfrak{X}_{t}$. Since $P_{\mathfrak{X}} \mathfrak{X}_{t}$ contains isometric copies of $\mathfrak{X}$ and all $\mathfrak{X}_{t}$, if $P_{\mathfrak{X}} \mathfrak{X}_{t}$ is to be $B$-convex, $\mathfrak{X}$ and all $\mathfrak{X}_{t}$ must be $k, \varepsilon$-convex for some common $k$ and $\varepsilon$. It is conjectured that this condition is sufficient. It also seems reasonable to conjecture that in Theorem II.17, the hypothesis that $\mathfrak{X}$ is uniformly convex can be weakened to state that $\mathfrak{X}$ is $2, \varepsilon$-convex and reach the same conclusion.

4. In Lemma I.4, we showed that if $A$ is closed under multiplication and $\mathfrak{X}$ is $A, k, \varepsilon$-convex, then $a_{n}(\mathfrak{X}, A) \leqq K n^{-\gamma}$ where the positive constants $K$ and $\gamma$ depend only on $k$ and $\varepsilon$. It would be of interest to know best possible bounds for $a_{n}(\mathfrak{X}, A)$ given that $\mathfrak{X}$ is $A, k, \varepsilon$-convex.

It is easy to show that given $\gamma>0$ there exists a $B$-convex (even uniformly convex) space $\mathfrak{X}$ (namely, $\ell_{p}$ for $p$ close enough to 1 ) such that $a_{n}(\mathfrak{X}, B) \neq O\left(n^{-\gamma}\right)$.

It would be of interest to know more about the constants $b_{n}(p, q, k, \varepsilon)$ of Theorem III.2. For example, for fixed $p, q, k$, and $\varepsilon$, how fast does $b_{n} \rightarrow 0$ ? For fixed $n$ what is the form of the dependence of $b_{n}$ on $p, q, k$, and $\varepsilon$ ?

5. ACKNOWLEDGEMENTS. This is essentially the author's Ph.D. thesis prepared under the direction of Professor Anatole Beck at the University of Wisconsin. The author wishes to express his gratitude to Professor Beck for the help and encouragement he gave during the course of this work. The author is also grateful to Professor Robert C. James of Harvey Mudd College for discussion and correspondence concerning his (James') work.

Research for and preparation of this paper were supported in part by the National Science Foundation (contract G-24335), the Air Force Office of Scientific Research (contract AF 49(638)-868) and the Office of Naval Research (contract NR 041-152). 


\title{
BIBLIOGRAPHY
}

1. Anatole Beck, $A$ convexity condition in Banach spaces and the strong law of large numbers, Proc. Amer. Math. Soc. 13 (1962), 329-334.

2. - On the strong law of large numbers, Ergodic Theory, Academic Press, New York, 1963.

3. James A. Clarkson, Uniformly convex spaces, Trans. Amer. Math. Soc. 40 (1936), 396-414.

4. M. M. Day, Some more uniformly convex spaces, Bull. Amer. Math. Soc. 47 (1941), 504-507.

5. —_ Uniform convexity. III, Bull. Amer. Math. Soc. 49 (1943), 745-750.

6. —_, Normed linear spaces, Academic Press, New York, 1962.

7. Nelson Dunford and J. T. Schwartz, Linear operators, Part I, Interscience, New York, 1958.

8. H. G. Eggleston, Convexity, Cambridge Univ. Press, Cambridge, 1958.

9. William Feller, An introduction to probability theory and its applications, Vol. I, Wiley, New York, 1957.

10. Daniel P. Giesy, A study of convexity in normed linear spaces, Ph.D. thesis, Univ. of Wisconsin, Madison, 1964.

11. R. C. James, Bases and reflexivity of Banach spaces, Ann. of Math. (2) 52 (1950), 518-522.

12. - A non-reflexive Banach space isometric with its second conjugate, Proc. Nat. Acad. Sci. U.S.A. 37 (1951), 174-177.

13. - - Uniformly non-square Banach spaces, Ann. of Math. 80 (1964), 542-550.

14. P. Jordan and J. von Neumann, On inner products in linear metric spaces, Ann. of Math. (2) 36 (1935), 719-723.

15. Michel Loève, Probability theory, Van Nostrand, New York, 1963.

16. A. R. Lovaglia, Locally uniformly convex Banach spaces, Trans. Amer. Math. Soc. 78 (1955), 225-238.

\author{
UNIVERSITY OF WISCONSIN, \\ MADISON, WISCONSIN \\ UNIVERSITY OF SOUTHERN CALIFORNIA, \\ Los ANGeles, California
}

